\title{
JACOBI RATIONAL APPROXIMATION AND SPECTRAL METHOD FOR DIFFERENTIAL EQUATIONS OF DEGENERATE TYPE
}

\author{
ZHONG-QING WANG AND BEN-YU GUO
}

\begin{abstract}
We introduce an orthogonal system on the half line, induced by Jacobi polynomials. Some results on the Jacobi rational approximation are established, which play important roles in designing and analyzing the Jacobi rational spectral method for various differential equations, with the coefficients degenerating at certain points and growing up at infinity. The Jacobi rational spectral method is proposed for a model problem appearing frequently in finance. Its convergence is proved. Numerical results demonstrate the efficiency of this new approach.
\end{abstract}

\section{INTRODUCTION}

Many problems arising in fluid dynamics, quantum mechanics, astrophysics, financial mathematics and other fields are set in unbounded domains. Several spectral methods have been developed for solving such problems. The first method is to use the Hermite and Laguerre approximations. In the second method, we reformulate original problems on unbounded domains to certain singular problems on bounded domains and then use the Jacobi spectral method for the resulting equations. Another effective method is based on rational approximations, induced by Legendre or Chebyshev polynomials; see [6, 7, 11, 13, 14, 17, 18, 22, 23. By using this approach, we can approximate differential equations on unbounded domains directly, without any artificial boundary and variable transformation. However, it does not work well sometimes. Indeed, the rational functions used in the past work are induced only by Legendre or Chebyshev polynomials. Accordingly, the weight functions of the corresponding orthogonal systems are fixed, which are not appropriate in many cases. This drawback limits the applications of the rational spectral method seriously. For instance, we consider the equation

$$
\begin{gathered}
\partial_{t}^{m} U(x, t)-\partial_{x}\left(a(x, t) \partial_{x} U(x, t)\right)-b(x, t) \partial_{x} U(x, t)+c(x, t) U(x, t)=F(x, t), \\
0<x<\infty, t>0
\end{gathered}
$$

Received by the editor March 15, 2006 and, in revised form, February 14, 2007.

2000 Mathematics Subject Classification. Primary 41A20, 65M70, 35K65.

Key words and phrases. Jacobi rational approximation, spectral method for differential equations of degenerate type on the half line, applications.

The work of the authors was partially supported by NSF of China, N.10471095 and N.10771142, the National Basic Research Project No. 2005CB321701, SF of Shanghai, N.04JC14062, The Fund of Chinese Education Ministry, N.20040270002, Shanghai Leading Academic Discipline Project, N.T0401, and The Fund for E-institutes of Shanghai Universities, N.E03004.

(C)2007 American Mathematical Society Reverts to public domain 28 years from publication 
where $m=1$ or 2 , and the coefficients $a(x, t), b(x, t)$ and $c(x, t)$ degenerate at $x=0$ and grow up at infinity. These equations come from some important problems, such as the spherically symmetrical waves in fluid dynamics and quantum mechanics, the degenerate parabolic equations in financial mathematics and exterior problems; see, e.g., 2, 4, 20, 21. Since in these cases, the coefficients of leading terms of differential equations degenerate or grow up at certain points, the existing rational spectral methods are no longer available. On the other hand, the analysis in the existing literatures concerning the Legendre and Chebyshev rational approximations was carried out in a twisted way. Consequently, the results are not optimal even for regular differential equations.

In this paper, we investigate the Jacobi rational approximation and its applications. The key points are as follows. Firstly, the base functions used in actual computation are induced by the general Jacobi polynomials with two parameters. By adjusting these parameters properly, the related systems of rational functions are mutually orthogonal associated with the weight functions which are exactly the same as in the underlying problems. This enlarges the applications of spectral methods essentially. Next, we deal with the rational approximation in a direct way. This leads to a series of optimal approximation results as the mathematical foundation of various spectral methods for the half line and other related problems. It also provides a powerful framework for the analysis of rational approximation. Finally, as an example, we propose the Jacobi rational spectral method for an important model problem. The numerical results demonstrate its high accuracy. We also discuss the applications of the proposed method to many other problems.

This paper is organized as follows. In the next section, we establish the basic results on the Jacobi rational approximations in nonuniformly weighted Sobolev spaces. In section 3, we propose the Jacobi rational spectral scheme for a model problem and prove its convergence. We also explore other applications. In section 4, we present some numerical results. The final section is for concluding remarks.

\section{JACOBI RATIONAL APPROXIMATION}

In this section, we develop the Jacobi rational approximation.

2.1. Jacobi polynomials. We first recall some properties of Jacobi polynomials. Let $I=\{y|| y \mid<1\}$. The Jacobi polynomials $J_{l}^{(\alpha, \beta)}(y), l=0,1,2, \cdots$, are the eigenfunctions of the Sturm-Liouville problem

$$
\partial_{y}\left((1-y)^{\alpha+1}(1+y)^{\beta+1} \partial_{y} v(y)\right)+\lambda(1-y)^{\alpha}(1+y)^{\beta} v(y)=0, \quad y \in I .
$$

The corresponding eigenvalues are $\lambda_{l}^{(\alpha, \beta)}=l(l+\alpha+\beta+1), l=0,1,2, \cdots$.

Let $\Gamma(x)$ be the Gamma function. It is noted that

$$
J_{l}^{(\alpha, \beta)}(-y)=(-1)^{l} J_{l}^{(\beta, \alpha)}(y), \quad J_{l}^{(\alpha, \beta)}(1)=\frac{\Gamma(l+\alpha+1)}{l ! \Gamma(\alpha+1)} .
$$

The Jacobi polynomials fulfill the recurrence relations (see [1])

$$
\begin{aligned}
2(l+ & \alpha+1) J_{l}^{(\alpha, \beta)}(y)-2(l+1) J_{l+1}^{(\alpha, \beta)}(y) \\
& =(2 l+\alpha+\beta+2)(1-y)^{-1} J_{l}^{(\alpha+1, \beta)}(y),
\end{aligned}
$$




$$
\begin{gathered}
J_{l}^{(\alpha, \beta-1)}(y)-J_{l}^{(\alpha-1, \beta)}(y)=J_{l-1}^{(\alpha, \beta)}(y), \\
(l+\alpha+\beta) J_{l}^{(\alpha, \beta)}(y)=(l+\beta) J_{l}^{(\alpha, \beta-1)}(y)+(l+\alpha) J_{l}^{(\alpha-1, \beta)}(y),
\end{gathered}
$$

and

$$
\partial_{y} J_{l}^{(\alpha, \beta)}(y)=\frac{1}{2}(l+\alpha+\beta+1) J_{l-1}^{(\alpha+1, \beta+1)}(y) .
$$

Let $\chi^{(\alpha, \beta)}(y)=(1-y)^{\alpha}(1+y)^{\beta}$. For $\alpha, \beta>-1$, the set of Jacobi polynomials is a complete $L_{\chi^{(\alpha, \beta)}}^{2}(I)$-orthogonal system, i.e.,

$$
\int_{\Lambda} J_{l}^{(\alpha, \beta)}(y) J_{l^{\prime}}^{(\alpha, \beta)}(y) \chi^{(\alpha, \beta)}(y) d y=\eta_{l}^{(\alpha, \beta)} \delta_{l, l^{\prime}}
$$

where $\delta_{l, l^{\prime}}$ is the Kronecker function and

$$
\eta_{l}^{(\alpha, \beta)}=\frac{2^{\alpha+\beta+1} \Gamma(l+\alpha+1) \Gamma(l+\beta+1)}{(2 l+\alpha+\beta+1) \Gamma(l+1) \Gamma(l+\alpha+\beta+1)} .
$$

In the forthcoming discussions, we denote the norm and semi-norm of the weighted Sobolev space $H_{\chi^{(\alpha, \beta)}}^{r}(I)$ by $\|v\|_{r, \chi^{(\alpha, \beta), I}}$ and $|v|_{r, \chi^{(\alpha, \beta)}, I}$, respectively. In particular, $L_{\chi^{(\alpha, \beta)}}^{2}(I)=H_{\chi^{(\alpha, \beta)}}^{0}(I)$ and $\|v\|_{\chi^{(\alpha, \beta)}, I}=\|v\|_{0, \chi^{(\alpha, \beta)}, I}$.

2.2. Jacobi rational functions. We now introduce the new orthogonal system of rational functions induced by Jacobi polynomials.

Let $\Lambda=(0, \infty)$ and let $\chi(x)$ be a certain weight function. Denote by $\mathbb{N}$ the set of all nonnegative integers. For any $r \in \mathbb{N}$, we define the weighted Sobolev space $H_{\chi}^{r}(\Lambda)$ in the usual way and denote its inner product, semi-norm and norm by $(u, v)_{r, \chi},|v|_{r, \chi}$ and $\|v\|_{r, \chi}$, respectively. In particular, $L_{\chi}^{2}(\Lambda)=H_{\chi}^{0}(\Lambda),(u, v)_{\chi}=$ $(u, v)_{0, \chi}$ and $\|v\|_{\chi}=\|v\|_{0, \chi}$. For any $r>0$, we define $H_{\chi}^{r}(\Lambda)$ and its norms by space interpolation. The space $H_{0, \chi}^{r}(\Lambda)$ stands for the closure in $H_{\chi}^{r}(\Lambda)$ of the set $\mathcal{D}(\Lambda)$ consisting of all infinitely differentiable functions with compact support in $\Lambda$. When $\chi(x) \equiv 1$, we omit the subscript $\chi$ in the notation.

The Jacobi rational functions are given by

$$
R_{l}^{(\alpha, \beta)}(x)=J_{l}^{(\alpha, \beta)}\left(\frac{x-1}{x+1}\right), \quad l=0,1,2, \cdots .
$$

According to (2.1), $R_{l}^{(\alpha, \beta)}(x)$ are the eigenfunctions of the Sturm-Liouville problem

$$
\partial_{x}\left(x^{\beta+1}(x+1)^{-\alpha-\beta} \partial_{x} v(x)\right)+\lambda x^{\beta}(x+1)^{-\alpha-\beta-2} v(x)=0, \quad x \in \Lambda .
$$

The corresponding eigenvalues are $\lambda_{l}^{(\alpha, \beta)}=l(l+\alpha+\beta+1), l=0,1,2, \cdots$. Moreover, the recurrence relations (2.2) $-(2.6)$ imply that

$$
\begin{aligned}
& R_{l}^{(\alpha, \beta)}(x)=(-1)^{l} R_{l}^{(\beta, \alpha)}\left(\frac{1}{x}\right), \quad R_{l}^{(\alpha, \beta)}(\infty)=\frac{\Gamma(l+\alpha+1)}{l ! \Gamma(\alpha+1)}, \\
& (l+\alpha+1) R_{l}^{(\alpha, \beta)}(x)-(l+1) R_{l+1}^{(\alpha, \beta)}(x) \\
& \quad=(2 l+\alpha+\beta+2)(x+1)^{-1} R_{l}^{(\alpha+1, \beta)}(x), \\
& R_{l}^{(\alpha, \beta-1)}(x)-R_{l}^{(\alpha-1, \beta)}(x)=R_{l-1}^{(\alpha, \beta)}(x), \\
& (l+\alpha+\beta) R_{l}^{(\alpha, \beta)}(x)=(l+\beta) R_{l}^{(\alpha, \beta-1)}(x)+(l+\alpha) R_{l}^{(\alpha-1, \beta)}(x),
\end{aligned}
$$

and

$$
\partial_{x} R_{l}^{(\alpha, \beta)}(x)=(l+\alpha+\beta+1)(x+1)^{-2} R_{l-1}^{(\alpha+1, \beta+1)}(x), \quad l \geq 1 .
$$


Let $\chi_{R}^{(\alpha, \beta)}(x)=x^{\beta}(x+1)^{-\alpha-\beta-2}, \alpha, \beta>-1$. Thanks to (2.7) and (2.8), the Jacobi rational functions form a complete $L_{\chi_{R}^{(\alpha, \beta)}}^{2}(\Lambda)$-orthogonal system, i.e.,

$$
\int_{\Lambda} R_{l}^{(\alpha, \beta)}(x) R_{l^{\prime}}^{(\alpha, \beta)}(x) \chi_{R}^{(\alpha, \beta)}(x) d x=\gamma_{l}^{(\alpha, \beta)} \delta_{l, l^{\prime}}
$$

where

$$
\gamma_{l}^{(\alpha, \beta)}=\frac{\Gamma(l+\alpha+1) \Gamma(l+\beta+1)}{(2 l+\alpha+\beta+1) \Gamma(l+1) \Gamma(l+\alpha+\beta+1)} .
$$

For any $v \in L_{\chi_{R}^{(\alpha, \beta)}}^{2}(\Lambda)$,

$$
v(x)=\sum_{l=0}^{\infty} \hat{v}_{l}^{(\alpha, \beta)} R_{l}^{(\alpha, \beta)}(x), \quad \hat{v}_{l}^{(\alpha, \beta)}=\left(\gamma_{l}^{(\alpha, \beta)}\right)^{-1} \int_{\Lambda} v(x) R_{l}^{(\alpha, \beta)}(x) \chi_{R}^{(\alpha, \beta)}(x) d x .
$$

Now, for any $N \in \mathbb{N}, \mathcal{R}_{N}$ stands for the set of all Jacobi rational functions of degree at most $N$. Moreover, ${ }_{0} \mathcal{R}_{N}=\left\{v \mid v \in \mathcal{R}_{N}, v(0)=0\right\}$ and $\mathcal{R}_{N}^{0}=\{v \mid v \in$ $\left.\mathcal{R}_{N}, v(0)=v(\infty)=0\right\}$.

We next derive an inverse inequality and an embedding inequality which will be used in the analysis of the Jacobi rational approximation and its applications. Denote by $c$ a generic positive constant independent of any function and $N$.

Theorem 2.1. For any $\phi \in \mathcal{R}_{N}$ and $1 \leq p \leq q \leq \infty$,

$$
\|\phi\|_{L_{\chi_{R}^{(\alpha, \beta)}}^{q(\Lambda)}} \leq c N^{\sigma(\alpha, \beta)\left(\frac{1}{p}-\frac{1}{q}\right)}\|\phi\|_{L_{R}^{p}(\alpha, \beta)}(\Lambda)
$$

where $\sigma(\alpha, \beta)=2 \max (\alpha, \beta)+2$, if $\max (\alpha, \beta) \geq-\frac{1}{2}$, and $\sigma(\alpha, \beta)=1$, otherwise.

Proof. Let $y \in I$ and $x=\frac{1+y}{1-y}$. Denote by $\mathcal{P}_{N}$ the set of all algebraic polynomials of degree at most $N$. For any $\phi \in \mathcal{R}_{N}$, we set $\psi(y)=\phi\left(\frac{1+y}{1-y}\right)$. Clearly $\psi(y) \in \mathcal{P}_{N}$. By an inverse inequality in $\mathcal{P}_{N}$ (see Theorem 2.1 of [10]), for any $\psi \in \mathcal{P}_{N}$ and $1 \leq p \leq q \leq \infty$,

$$
\left(\int_{I}|\psi(y)|^{q} \chi^{(\alpha, \beta)}(y) d y\right)^{\frac{1}{q}} \leq c N^{\sigma(\alpha, \beta)\left(\frac{1}{p}-\frac{1}{q}\right)}\left(\int_{I}|\psi(y)|^{p} \chi^{(\alpha, \beta)}(y) d y\right)^{\frac{1}{p}} .
$$

It can be checked that

$$
\chi_{R}^{(\alpha, \beta)}(x)=2^{-\alpha-\beta-2}(1-y)^{\alpha+2}(1+y)^{\beta}, \quad \frac{d x}{d y}=\frac{2}{(1-y)^{2}} .
$$

Therefore

$$
\begin{aligned}
& \|\phi\|_{L_{\chi_{R}^{q}(\alpha, \beta)}^{q}(\Lambda)}^{q}=2^{-\alpha-\beta-1} \int_{I}|\psi(y)|^{q} \chi^{(\alpha, \beta)}(y) d y \\
& \leq c N^{\sigma(\alpha, \beta)\left(\frac{q}{p}-1\right)}\left(\int_{I}|\psi(y)|^{p} \chi^{(\alpha, \beta)}(y) d y\right)^{\frac{q}{p}} \leq c N^{\sigma(\alpha, \beta)\left(\frac{q}{p}-1\right)}\|\phi\|_{L_{\chi_{R}^{p}}^{p}(\alpha, \beta)}^{q}(\Lambda) .
\end{aligned}
$$

Theorem 2.2. For any $\phi \in \mathcal{R}_{N}$ and $r \geq 0$,

$$
\|\phi\|_{r, \chi_{R}^{(\alpha, \beta)}} \leq c N^{2 r}\|\phi\|_{\chi_{R}^{(\alpha, \beta)}} .
$$

If, in addition, $\alpha, \beta>r-1$, then

$$
\|\phi\|_{r, \chi_{R}^{(\alpha, \beta)}} \leq c N^{r}\|\phi\|_{\chi_{R}^{(\alpha-r, \beta-r)}} .
$$


Proof. Let $y \in I, \mathcal{P}_{N}$ and $\psi(y)$ be the same as in the proof of the last theorem. According to an inverse inequality (see Theorem 2.2 of [10]), for any $\psi(y) \in \mathcal{P}_{N}$ and $r \in \mathbb{N}$,

$$
\|\psi\|_{r, \chi^{(\alpha, \beta), I}} \leq c N^{2 r}\|\psi\|_{\chi^{(\alpha, \beta)}, I} .
$$

In particular, if $\alpha, \beta>r-1$, then

$$
\|\psi\|_{r, \chi^{(\alpha, \beta), I}} \leq c N^{r}\|\psi\|_{\chi^{(\alpha-r, \beta-r), I}} .
$$

By induction,

$$
\partial_{x}^{k} \phi(x)=\sum_{j=1}^{k} C_{k, j}(1-y)^{k+j} \partial_{y}^{j} \psi(y)
$$

where $C_{k, j}$ are some constants. Hence we use (2.15) and (2.17) to obtain that

$$
\begin{aligned}
|\phi|_{k, \chi_{R}^{(\alpha, \beta)}}^{2} & \leq c \sum_{j=1}^{k} \int_{I}(1-y)^{2 k+2 j+\alpha}(1+y)^{\beta}\left(\partial_{y}^{j} \psi(y)\right)^{2} d y \\
& \leq c\|\psi\|_{k, \chi^{(\alpha, \beta)}, I}^{2} \leq c N^{4 k}\|\psi\|_{\chi^{(\alpha, \beta)}, I}^{2} \leq c N^{4 k}\|\phi\|_{\chi_{R}^{(\alpha, \beta)}}^{2} .
\end{aligned}
$$

Furthermore, if $\alpha, \beta>k-1$, then by (2.16) and (2.17),

$$
|\phi|_{k, \chi_{R}^{(\alpha, \beta)}}^{2} \leq c\|\psi\|_{k, \chi^{(\alpha, \beta), I}}^{2} \leq c N^{2 k}\|\psi\|_{\chi^{(\alpha-k, \beta-k), I}}^{2} \leq c N^{2 k}\|\phi\|_{\chi_{R}^{(\alpha-k, \beta-k)}}^{2} .
$$

The previous statements with space interpolation lead to the desired results.

2.3. Basic results on Jacobi rational approximation. We now turn to several orthogonal projections which are frequently used in the Jacobi rational spectral method.

We first consider the orthogonal projection $P_{N, \alpha, \beta}: L_{\chi_{R}^{(\alpha, \beta)}}^{2}(\Lambda) \rightarrow \mathcal{R}_{N}$. It is defined by

$$
\left(P_{N, \alpha, \beta} v-v, \phi\right)_{\chi_{R}^{(\alpha, \beta)}}=0, \quad \forall \phi \in \mathcal{R}_{N} .
$$

In order to present the approximation results precisely, we introduce the space $H_{\chi_{R}^{(\alpha, \beta)}, A}^{r}(\Lambda), r \in \mathbb{N}$, with the following semi-norm and norm:

$$
\begin{gathered}
|v|_{r, \chi_{R}^{(\alpha, \beta)}, A}=\left(\sum_{l=r}^{\infty}\left(\lambda_{l}^{(\alpha, \beta)}\right)^{r}\left|\hat{v}_{l}^{(\alpha, \beta)}\right|^{2} \gamma_{l}^{(\alpha, \beta)}\right)^{\frac{1}{2}}, \\
\|v\|_{r, \chi_{R}^{(\alpha, \beta)}, A}=\left(\sum_{k=0}^{r}|v|_{k, \chi_{R}^{(\alpha, \beta)}, A}^{2}\right)^{\frac{1}{2}} .
\end{gathered}
$$

For any $r>0$, we define the space $H_{\chi_{R}^{(\alpha, \beta)}, A}^{r}(\Lambda)$ and its norm by space interpolation as in [3].

Lemma 2.1. For any $v \in H_{\chi_{R}^{(\alpha, \beta)}, A}^{r}(\Lambda), r \in \mathbb{N}$ and $0 \leq \mu \leq r$,

$$
\left\|P_{N, \alpha, \beta} v-v\right\|_{\mu, \chi_{R}^{(\alpha, \beta)}, A} \leq c_{0}\left(\lambda_{N+1}^{(\alpha, \beta)}\right)^{\frac{\mu-r}{2}}|v|_{r, \chi_{R}^{(\alpha, \beta)}, A}
$$

where $c_{0}=\left(1+\left(1-\left(\lambda_{N+1}^{(\alpha, \beta)}\right)^{-\mu}\right)\left(\lambda_{N+1}^{(\alpha, \beta)}-1\right)^{-1}\right)^{\frac{1}{2}} \approx 1$. 
Proof. We have that for nonnegative integers $k \leq \mu$,

$$
\begin{aligned}
\left|P_{N, \alpha, \beta} v-v\right|_{k, \chi_{R}^{(\alpha, \beta)}, A}^{2} & =\left|\sum_{l=N+1}^{\infty} \hat{v}_{l}^{(\alpha, \beta)} R_{l}^{(\alpha, \beta)}(x)\right|_{k, \chi_{R}^{(\alpha, \beta)}, A}^{2} \\
& \leq \sum_{l=N+1}^{\infty}\left(\lambda_{l}^{(\alpha, \beta)}\right)^{k}\left|\hat{v}_{l}^{(\alpha, \beta)}\right|^{2} \gamma_{l}^{(\alpha, \beta)} \leq\left(\lambda_{N+1}^{(\alpha, \beta)}\right)^{k-r}|v|_{r, \chi_{R}^{(\alpha, \beta)}, A}^{2} .
\end{aligned}
$$

Consequently,

$$
\begin{aligned}
& \left\|P_{N, \alpha, \beta} v-v\right\|_{\mu, \chi_{R}^{(\alpha, \beta)}, A}^{2}=\sum_{k=0}^{\mu}\left|P_{N, \alpha, \beta} v-v\right|_{k, \chi_{R}^{(\alpha, \beta)}, A}^{2} \\
& \leq\left(\lambda_{N+1}^{(\alpha, \beta)}\right)^{-r}|v|_{r, \chi_{R}^{(\alpha, \beta)}, A}^{2} \sum_{k=0}^{\mu}\left(\lambda_{N+1}^{(\alpha, \beta)}\right)^{k}=c_{0}^{2}\left(\lambda_{N+1}^{(\alpha, \beta)}\right)^{\mu-r}|v|_{r, \chi_{R}^{(\alpha, \beta)}, A}^{2} .
\end{aligned}
$$

For any $\mu=[\mu]+\theta, 0<\theta<1$, we use the Gagliardo-Nirenberg inequality (cf. [3]) to reach that

$$
\begin{aligned}
\left\|P_{N, \alpha, \beta} v-v\right\|_{\mu, \chi_{R}^{(\alpha, \beta)}, A} & \leq\left\|P_{N, \alpha, \beta} v-v\right\|_{[\mu], \chi_{R}^{(\alpha, \beta)}, A}^{1-\theta}\left\|P_{N, \alpha, \beta} v-v\right\|_{[\mu]+1, \chi_{R}^{(\alpha, \beta)}, A}^{\theta} \\
& \leq c_{0}\left(\lambda_{N+1}^{(\alpha, \beta)}\right)^{\frac{\mu-r}{2}}|v|_{r, \chi_{R}^{(\alpha, \beta)}, A} .
\end{aligned}
$$

Remark 2.1. Letting any $\phi \in \mathcal{R}_{N}$ and replacing $v$ by $\phi-v$ in (2.20), we deduce that

$$
\left\|P_{N, \alpha, \beta} v-v\right\|_{\mu, \chi_{R}^{(\alpha, \beta)}, A} \leq c_{0}\left(\lambda_{N+1}^{(\alpha, \beta)}\right)^{\frac{\mu-r}{2}} \inf _{\phi \in \mathcal{R}_{N}}|\phi-v|_{r, \chi_{R}^{(\alpha, \beta)}, A} .
$$

On the right side of (2.20), the semi-norm $|v|_{r, \chi_{R}^{(\alpha, \beta)}, A}$ is given by (2.19). Accordingly, we used it to derive the basic result (2.20) easily. But it is not convenient for its applications. Fortunately, there exists an equivalent representation for such semi-norm for any $r \in \mathbb{N}$. To show this, let

$$
R_{l, 0}^{(\alpha, \beta)}(x)=R_{l}^{(\alpha, \beta)}(x), \quad R_{l, k}^{(\alpha, \beta)}(x)=(x+1)^{2} \partial_{x} R_{l, k-1}^{(\alpha, \beta)}(x), \quad k \geq 1 .
$$

With the aid of (2.11) and (2.22), we can use induction to show that

$$
R_{l, k}^{(\alpha, \beta)}(x)=\frac{\Gamma(l+\alpha+\beta+k+1)}{\Gamma(l+\alpha+\beta+1)} R_{l-k}^{(\alpha+k, \beta+k)}(x), \quad l \geq k .
$$

Thus $R_{l, k}^{(\alpha, \beta)}(x)$ is the same as $R_{l-k}^{(\alpha+k, \beta+k)}(x)$, apart from a constant. Thus, by (2.9),

$$
\begin{aligned}
& \partial_{x}\left(x^{\beta+k+1}(x+1)^{-\alpha-\beta-2 k} \partial_{x} R_{l, k}^{(\alpha, \beta)}(x)\right)+\lambda_{l-k}^{(\alpha+k, \beta+k)} x^{\beta+k}(x+1)^{-\alpha-\beta-2 k-2} R_{l, k}^{(\alpha, \beta)}(x) \\
& \quad=0 .
\end{aligned}
$$

Multiplying the above by $R_{l, k}^{(\alpha, \beta)}(x)$ and integrating the result by parts, we assert that

$$
\left\|\partial_{x} R_{l, k}^{(\alpha, \beta)}\right\|_{\chi_{R}^{(\alpha+k-3, \beta+k+1)}}^{2}=\lambda_{l-k}^{(\alpha+k, \beta+k)}\left\|R_{l, k}^{(\alpha, \beta)}\right\|_{\chi_{R}^{(\alpha+k, \beta+k)}}^{2} .
$$

Furthermore, a combination of (2.22) and (2.24) gives that

$$
\begin{aligned}
& \left\|R_{l, k}^{(\alpha, \beta)}\right\|_{\chi_{R}^{(\alpha+k, \beta+k)}}^{2}=\left\|\partial_{x} R_{l, k-1}^{(\alpha, \beta)}\right\|_{\chi_{R}^{(\alpha+k-4, \beta+k)}}^{2} \\
& =\lambda_{l-k+1}^{(\alpha+k-1, \beta+k-1)}\left\|R_{l, k-1}^{(\alpha, \beta)}\right\|_{\chi_{R}^{(\alpha+k-1, \beta+k-1)}}^{2}=\cdots=c_{l, k}^{(\alpha, \beta)} \gamma_{l}^{(\alpha, \beta)}
\end{aligned}
$$


where

$$
c_{l, k}^{(\alpha, \beta)}=\prod_{j=0}^{k-1} \lambda_{l-j}^{(\alpha+j, \beta+j)}=\prod_{j=0}^{k-1}(l-j)(l+j+\alpha+\beta+1) .
$$

For simplicity of statements, we introduce the notation

$$
d_{l, k}^{(\alpha, \beta)}=c_{l, k}^{(\alpha, \beta)}\left(\lambda_{l}^{(\alpha, \beta)}\right)^{-k}=\prod_{j=0}^{k-1}\left(1-\frac{j}{l}\right)\left(1+\frac{j}{l+\alpha+\beta+1}\right) .
$$

Then we have

$$
\begin{aligned}
& \left(1-\frac{k-1}{l}\right)^{k-1} \leq \prod_{j=0}^{k-1}\left(1-\frac{j}{l}\right) \leq d_{l, k}^{(\alpha, \beta)} \\
& \leq \prod_{j=0}^{k-1}\left(1+\frac{j}{l+\alpha+\beta+1}\right) \leq\left(1+\frac{k-1}{l+\alpha+\beta+1}\right)^{k-1} .
\end{aligned}
$$

Thereby, for $l \gg k$,

$$
d_{0} \leq d_{l, k}^{(\alpha, \beta)} \leq d_{1}, \quad d_{0}, d_{1} \simeq 1
$$

Next, we define

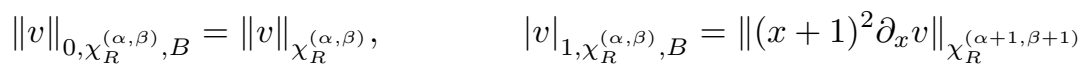

and

$$
|v|_{k, \chi_{R}^{(\alpha, \beta)}, B}=\left|(x+1)^{2} \partial_{x} v\right|_{k-1, \chi_{R}^{(\alpha+1, \beta+1)}, B}, \quad k \geq 2 .
$$

We shall show that $|v|_{k, \chi_{R}^{(\alpha, \beta)}, B}$ and $|v|_{k, \chi_{R}^{(\alpha, \beta)}, A}$ are equivalent noms. To do this, we set

$$
g_{1}(v)=(x+1)^{2} \partial_{x} v(x), \quad g_{k}(v)=(x+1)^{2} \partial_{x}\left(g_{k-1}(v)\right), \quad k \geq 1 .
$$

By (2.23),$\partial_{x} R_{k, k}^{(\alpha, \beta)}(x)=0$. Thus, we use (2.14), (2.22) and induction to verify that

$$
g_{k}(v)=\sum_{l=k}^{\infty} \hat{v}_{l}^{(\alpha, \beta)} R_{l, k}^{(\alpha, \beta)}(x) .
$$

Therefore, with the aid of (2.25), (2.27)-(2.29) and the definition of $d_{l, k}^{(\alpha, \beta)}$, we verify that

$$
\begin{aligned}
|v|_{k, \chi_{R}^{(\alpha, \beta)}, B}^{2} & =\left|(x+1)^{2} \partial_{x} v\right|_{k-1, \chi_{R}^{(\alpha+1, \beta+1)}, B}^{2}=\cdots=\left\|g_{k}(v)\right\|_{\chi_{R}^{(\alpha+k, \beta+k)}}^{2} \\
& =\sum_{l=k}^{\infty}\left|\hat{v}_{l}^{(\alpha, \beta)}\right|^{2}\left\|R_{l, k}^{(\alpha, \beta)}\right\|_{\chi_{R}^{(\alpha+k, \beta+k)}}^{2}=\sum_{l=k}^{\infty} d_{l, k}^{(\alpha, \beta)}\left(\lambda_{l}^{(\alpha, \beta)}\right)^{k}\left|\hat{v}_{l}^{(\alpha, \beta)}\right|^{2} \gamma_{l}^{(\alpha, \beta)} .
\end{aligned}
$$

In view of this fact, we use (2.19) and (2.26) to obtain that for all $k \in \mathbb{N}$,

$$
c d_{0}|v|_{k, \chi_{R}^{(\alpha, \beta)}, A}^{2} \leq|v|_{k, \chi_{R}^{(\alpha, \beta)}, B}^{2} \leq c d_{1}|v|_{k, \chi_{R}^{(\alpha, \beta)}, A}^{2} .
$$

A combination of (2.19) and (2.30) implies that

$$
c \sqrt{d_{0}}\|v\|_{r, \chi_{R}^{(\alpha, \beta)}, A} \leq\left(\sum_{k=0}^{r}|v|_{k, \chi_{R}^{(\alpha, \beta)}, B}^{2}\right)^{\frac{1}{2}} \leq c \sqrt{d_{1}}\|v\|_{r, \chi_{R}^{(\alpha, \beta)}, A} .
$$


According to the previous statements, we now redefine the space $H_{\chi_{R}^{(\alpha, \beta)}, A}^{r}(\Lambda)$ as

$$
H_{\chi_{R}^{(\alpha, \beta)}, A}^{r}(\Lambda)=\left\{v \mid v \text { is measurable and }\|v\|_{r, \chi_{R}^{(\alpha, \beta)}, A}<\infty\right\}, \quad r \in \mathbb{N},
$$

equipped with the following semi-norm and norm:

$$
\begin{aligned}
& \|v\|_{0, \chi_{R}^{(\alpha, \beta)}, A}=\|v\|_{\chi_{R}^{(\alpha, \beta)},}, \quad|v|_{1, \chi_{R}^{(\alpha, \beta)}, A}=\left\|(x+1)^{2} \partial_{x} v\right\|_{\chi_{R}^{(\alpha+1, \beta+1)}}, \\
& |v|_{k, \chi_{R}^{(\alpha, \beta)}, A}=\left|(x+1)^{2} \partial_{x} v\right|_{k-1, \chi_{R}^{(\alpha+1, \beta+1)}, A}, \quad k \geq 2, \\
& \|v\|_{r, \chi_{R}^{(\alpha, \beta)}, A}=\left(\sum_{k=0}^{r}|v|_{k, \chi_{R}^{(\alpha, \beta)}, A}^{2}\right)^{\frac{1}{2}} .
\end{aligned}
$$

For any $r>0$, we define the space $H_{\chi_{R}^{(\alpha, \beta)}, A}^{r}(\Lambda)$ and its norm by space interpolation as in $[3]$.

As a direct result of Lemma 2.1 and the above statements, we have the following result.

Theorem 2.3. For any $v \in H_{\chi_{R}^{(\alpha, \beta)}, A}^{r}(\Lambda), r \in \mathbb{N}$ and $0 \leq \mu \leq r$,

$$
\left\|P_{N, \alpha, \beta} v-v\right\|_{\mu, \chi_{R}^{(\alpha, \beta)}, A} \leq c N^{\mu-r}|v|_{r, \chi_{R}^{(\alpha, \beta)}, A} .
$$

2.4. Orthogonal projection in nonuniformly weighted space. In many practical problems, the coefficients of terms involving derivatives of different orders might degenerate or grow up in different ways. In these cases, it is impossible to compare numerical solutions with exact solutions in the usual Sobolev spaces, whereas it might be carried out in certain nonuniformly weighted Sobolev spaces which the exact solutions belong to; see, e.g., 10, 16]. It is also true for the Jacobi rational approximation. To do this, we introduce the space $H_{\alpha, \beta, \gamma, \delta}^{\mu}(\Lambda), 0 \leq \mu \leq 1$, with the norm $\|v\|_{\mu, \alpha, \beta, \gamma, \delta}$. For $\mu=0, H_{\alpha, \beta, \gamma, \delta}^{0}(\Lambda)=L_{\chi_{R}^{(\gamma, \delta)}}^{2}(\Lambda)$. For $\mu=1$,

$$
H_{\alpha, \beta, \gamma, \delta}^{1}(\Lambda)=\left\{v \mid v \text { is measurable and }\|v\|_{1, \alpha, \beta, \gamma, \delta}<\infty\right\}
$$

with the norm

$$
\|v\|_{1, \alpha, \beta, \gamma, \delta}=\left(|v|_{1, \chi_{R}^{(\alpha, \beta)}}^{2}+\|v\|_{\chi_{R}^{(\gamma, \delta)}}^{2}\right)^{\frac{1}{2}} .
$$

For $0<\mu<1$, the space $H_{\alpha, \beta, \gamma, \delta}^{\mu}(\Lambda)$ and its norm are defined by space interpolation.

Now, let

$$
a_{\alpha, \beta, \gamma, \delta}(u, v)=\left(\partial_{x} u, \partial_{x} v\right)_{\chi_{R}^{(\alpha, \beta)}}+(u, v)_{\chi_{R}^{(\gamma, \delta)}}, \forall u, v \in H_{\alpha, \beta, \gamma, \delta}^{1}(\Lambda) .
$$

The orthogonal projection $P_{N, \alpha, \beta, \gamma, \delta}^{1}: H_{\alpha, \beta, \gamma, \delta}^{1}(\Lambda) \rightarrow \mathcal{R}_{N}$ is defined by

$$
a_{\alpha, \beta, \gamma, \delta}\left(P_{N, \alpha, \beta, \gamma, \delta}^{1} v-v, \phi\right)=0, \forall \phi \in \mathcal{R}_{N} .
$$

The following imbedding inequality will be used for the derivation of approximation results.

Lemma 2.2. If

$$
\alpha \leq \gamma-2, \quad \beta \leq \delta+2, \quad \gamma, \delta>-1,
$$

then for any $v \in H_{\chi_{R}^{(\alpha, \beta)}}^{1}(\Lambda)$ with $v(1)=0$,

$$
\|v\|_{\chi_{R}^{(\gamma, \delta)}} \leq c|v|_{1, \chi_{R}^{(\alpha, \beta)} .}
$$


Proof. Let $u(y)=v\left(\frac{1+y}{1-y}\right)$. Obviously $u(0)=0$. By Lemma 2.3 of $[10],\|u\|_{\chi^{(\gamma, \delta)}, I} \leq$ $c|u|_{1, \chi(\gamma+2, \delta+2), I}$. So, a simple calculation yields that

$$
\|v\|_{\chi_{R}^{(\gamma, \delta)}} \leq c\|u\|_{\chi^{(\gamma, \delta), I}} \leq c|u|_{1, \chi^{(\gamma+2, \delta+2), I}} \leq c|v|_{1, \chi_{R}^{(\gamma-2, \delta+2)}} \leq c|v|_{1, \chi_{R}^{(\alpha, \beta)}} .
$$

Theorem 2.4. Let $\sigma \leq 4$ and $\theta \leq 0$. If

$$
-1<\alpha+\sigma \leq \gamma+2, \quad-1<\beta+\theta \leq \delta+2, \quad \gamma, \delta>-1,
$$

then for any $v \in H_{\chi_{R}^{(\alpha+\sigma-1, \beta+\theta-1)}, A}^{r}(\Lambda), r \in \mathbb{N}$ and $r \geq 1$,

$$
\left\|P_{N, \alpha, \beta, \gamma, \delta}^{1} v-v\right\|_{1, \alpha, \beta, \gamma, \delta} \leq c N^{1-r}|v|_{r, \chi_{R}^{(\alpha+\sigma-1, \beta+\theta-1)}, A} .
$$

If, in addition,

$$
\alpha \leq \gamma+\sigma-7, \quad \beta \leq \delta+\theta+1,
$$

then for $0 \leq \mu \leq 1$,

$$
\left\|P_{N, \alpha, \beta, \gamma, \delta}^{1} v-v\right\|_{\mu, \alpha, \beta, \gamma, \delta} \leq c N^{\mu-r}|v|_{r, \chi_{R}^{(\alpha+\sigma-1, \beta+\theta-1)}, A} .
$$

Proof. We first prove (2.35). Let

$$
\phi(x)=\int_{0}^{x}(z+1)^{-2} P_{N-1, \alpha+\sigma, \beta+\theta}\left((z+1)^{2} \partial_{z} v(z)\right) d z+\xi
$$

where $\xi$ is chosen in such a way that $v(1)=\phi(1)$. By (2.11), there exists $\psi \in \mathcal{R}_{N}$ such that

$$
\phi(x)=\int_{0}^{x} \partial_{z} \psi(z) d z+\xi=\psi(x)-\psi(0)+\xi \in \mathcal{R}_{N} .
$$

Clearly, $|\phi-v|_{\chi_{R}^{(\alpha, \beta)}} \leq c|\phi-v|_{\chi_{R}^{(\alpha+\sigma-4, \beta+\theta)}}$. Consequently, we use the projection theorem, Lemma 2.2 and Theorem 2.3 to deduce that

$$
\begin{aligned}
& \left\|P_{N, \alpha, \beta, \gamma, \delta}^{1} v-v\right\|_{1, \alpha, \beta, \gamma, \delta} \leq\|\phi-v\|_{1, \alpha, \beta, \gamma, \delta} \leq c|\phi-v|_{1, \chi_{R}^{(\alpha+\sigma-4, \beta+\theta)}} \\
& \quad=c\left\|P_{N-1, \alpha+\sigma, \beta+\theta}\left((x+1)^{2} \partial_{x} v\right)-(x+1)^{2} \partial_{x} v\right\|_{\chi_{R}^{(\alpha+\sigma, \beta+\theta)}} \\
& \quad \leq c N^{1-r}|v|_{r, \chi_{R}^{(\alpha+\sigma-1, \beta+\theta-1)}, A} .
\end{aligned}
$$

We now prove (2.37) with condition (2.36). Let $g \in L_{\chi_{R}^{(\gamma, \delta)}}^{2}(\Lambda)$ and consider the problem

$$
a_{\alpha, \beta, \gamma, \delta}(w, z)=(g, z)_{\chi_{R}^{(\gamma, \delta)}}, \quad \forall z \in H_{\alpha, \beta, \gamma, \delta}^{1}(\Lambda) .
$$

Taking $z=w$ in (2.39), we get that $\|w\|_{1, \alpha, \beta, \gamma, \delta} \leq\|g\|_{\chi_{R}^{(\gamma, \delta)}}$.

We also need to estimate the upper-bounds of some weighted norms of $\partial_{x}^{2} w(x)$ and $\partial_{x} w(x)$.

First, let $z(x)$ vary in $\mathcal{D}(\Lambda)$, and so in the sense of distributions,

$$
-\partial_{x}\left(\partial_{x} w(x) \chi_{R}^{(\alpha, \beta)}(x)\right)=(g(x)-w(x)) \chi_{R}^{(\gamma, \delta)}(x) .
$$

Next, integrating (2.40) yields that

$$
\left|\partial_{x} w\left(x_{2}\right) \chi_{R}^{(\alpha, \beta)}\left(x_{2}\right)-\partial_{x} w\left(x_{1}\right) \chi_{R}^{(\alpha, \beta)}\left(x_{1}\right)\right| \leq\|g-w\|_{\chi_{R}^{(\gamma, \delta)}}\left(\int_{x_{1}}^{x_{2}} \chi_{R}^{(\gamma, \delta)}(x) d x\right)^{\frac{1}{2}} .
$$

Thus $\partial_{x} w(x) \chi_{R}^{(\alpha, \beta)}(x)$ is meaningful at $x=0, \infty$. Moreover, multiplying (2.40) by any $z \in H_{\alpha, \beta, \gamma, \delta}^{1}(\Lambda)$, integrating the resulting equality by parts and using (2.39), 
we obtain that

$$
\begin{array}{r}
\partial_{x} w(\infty) z(\infty) \chi_{R}^{(\alpha, \beta)}(\infty)-\partial_{x} w(0) z(0) \chi_{R}^{(\alpha, \beta)}(0) \\
=\int_{\Lambda}\left(\partial_{x} w(x) \partial_{x} z(x) \chi_{R}^{(\alpha, \beta)}(x)-(g(x)-w(x)) z(x) \chi_{R}^{(\gamma, \delta)}(x)\right) d x=0, \\
\forall z \in H_{\alpha, \beta, \gamma, \delta}^{1}(\Lambda) .
\end{array}
$$

Thereby, $\partial_{x} w(x) \chi_{R}^{(\alpha, \beta)}(x) \rightarrow 0$ as $x \rightarrow 0, \infty$.

Furthermore, we have from (2.40) that

$$
\begin{aligned}
-\partial_{x}^{2} w(x)= & \left(\beta x^{-1}-(\alpha+\beta+2)(x+1)^{-1}\right) \partial_{x} w(x) \\
& +(g(x)-w(x)) x^{\delta-\beta}(x+1)^{\alpha+\beta-\gamma-\delta} .
\end{aligned}
$$

Let $\Lambda_{1}=(0,1]$ and $\Lambda_{2}=(1, \infty)$. A direct calculation with (2.41) gives that

$$
\left\|\partial_{x}^{2} w x^{\frac{1+\theta}{2}}(x+1)^{3-\frac{\sigma+\theta}{2}}\right\|_{\chi_{R}^{(\alpha, \beta)}}^{2}+4\left\|\partial_{x} w x^{\frac{1+\theta}{2}}(x+1)^{2-\frac{\sigma+\theta}{2}}\right\|_{\chi_{R}^{(\alpha, \beta)}}^{2} \leq c\left(D_{1}+D_{2}\right)
$$

where $D_{1}=D_{1}\left(\Lambda_{1}\right)+D_{1}\left(\Lambda_{2}\right)$ and

$$
\begin{aligned}
& D_{1}\left(\Lambda_{j}\right)=\int_{\Lambda_{j}} x^{\beta+\theta-1}(x+1)^{-\alpha-\beta-\sigma-\theta+4}\left(\partial_{x} w(x)\right)^{2} d x, \quad j=1,2, \\
& D_{2}=\int_{\Lambda}(g(x)-w(x))^{2} x^{2 \delta-\beta+\theta+1}(x+1)^{\alpha+\beta-2 \gamma-2 \delta-\sigma-\theta+4} d x
\end{aligned}
$$

Obviously, (2.36) implies that

$$
D_{2}=\int_{\Lambda}(g(x)-w(x))^{2} \chi_{R}^{(\gamma, \delta)}\left(\frac{x}{x+1}\right)^{\delta-\beta+\theta+1}(x+1)^{\alpha-\gamma-\sigma+7} d x \leq\|g-w\|_{\chi_{R}^{(\gamma, \delta)}}^{2} .
$$

So it remains to estimate $D_{1}\left(\Lambda_{j}\right), j=1,2$. Due to (2.40),

$$
\begin{aligned}
\partial_{x} w(x) & =-x^{-\beta}(x+1)^{\alpha+\beta+2} \int_{0}^{x}(g(z)-w(z)) \chi_{R}^{(\gamma, \delta)}(z) d z \\
& =x^{-\beta}(x+1)^{\alpha+\beta+2} \int_{x}^{\infty}(g(z)-w(z)) \chi_{R}^{(\gamma, \delta)}(z) d z
\end{aligned}
$$

This fact, along with (2.36) and the Hardy inequality (see [19]) leads to (2.44)

$$
\begin{aligned}
D_{1}\left(\Lambda_{1}\right) & =\int_{0}^{1} x^{-\beta+\theta-1}(x+1)^{\alpha+\beta-\sigma-\theta+8}\left(\int_{0}^{x}(g(z)-w(z)) \chi_{R}^{(\gamma, \delta)}(z) d z\right)^{2} d x \\
& \leq c \int_{0}^{1} x^{-\delta-2}\left(\int_{0}^{x}(g(z)-w(z)) \chi_{R}^{(\gamma, \delta)}(z) d z\right)^{2} d x \\
& \leq c \int_{0}^{1} x^{-\delta}(g(x)-w(x))^{2}\left(\chi_{R}^{(\gamma, \delta)}(x)\right)^{2} d x \leq c\|g-w\|_{\chi_{R}^{(\gamma, \delta)}}^{2} .
\end{aligned}
$$

We can estimate $D_{1}\left(\Lambda_{2}\right)$ similarly. To do this, let $y=\frac{x-1}{x+1}, \xi=\frac{z-1}{z+1}, \tilde{g}(\xi)=g\left(\frac{1+\xi}{1-\xi}\right)$ and $\tilde{w}(\xi)=w\left(\frac{1+\xi}{1-\xi}\right)$. Then by virtue of (2.36), (2.43) and the Hardy inequality, we 
deduce that

$$
\begin{aligned}
& D_{1}\left(\Lambda_{2}\right)=\int_{1}^{\infty} x^{-\beta+\theta-1}(x+1)^{\alpha+\beta-\sigma-\theta+8}\left(\int_{x}^{\infty}(g(z)-w(z)) \chi_{R}^{(\gamma, \delta)}(z) d z\right)^{2} d x \\
& \leq c \int_{0}^{1}(1-y)^{-\alpha+\sigma-9}(1+y)^{-\beta+\theta-1}\left(\int_{y}^{1}(\tilde{g}(\xi)-\tilde{w}(\xi)) \chi^{(\gamma, \delta)}(\xi) d \xi\right)^{2} d y \\
& \leq c \int_{0}^{1}(1-y)^{-\gamma-2}\left(\int_{y}^{1}(\tilde{g}(\xi)-\tilde{w}(\xi)) \chi^{(\gamma, \delta)}(\xi) d \xi\right)^{2} d y \\
& \leq c \int_{0}^{1}(1-y)^{-\gamma}(\tilde{g}(y)-\tilde{w}(y))^{2}\left(\chi^{(\gamma, \delta)}(y)\right)^{2} d y \leq c \int_{0}^{1}(\tilde{g}(y)-\tilde{w}(y))^{2} \chi^{(\gamma, \delta)}(y) d y \\
& \leq c \int_{1}^{\infty}(g(x)-w(x))^{2} \chi_{R}^{(\gamma, \delta)}(x) d x \leq c\|g-w\|_{\chi_{R}^{(\gamma, \delta)}}^{2} .
\end{aligned}
$$

A combination of (2.42) and the previous estimates gives that

$$
\begin{aligned}
& \left\|\partial_{x}^{2} w x^{\frac{1+\theta}{2}}(x+1)^{3-\frac{\sigma+\theta}{2}}\right\|_{\chi_{R}^{(\alpha, \beta)}}^{2}+4\left\|\partial_{x} w x^{\frac{1+\theta}{2}}(x+1)^{2-\frac{\sigma+\theta}{2}}\right\|_{\chi_{R}^{(\alpha, \beta)}}^{2} \\
& \leq c\|g-w\|_{\chi_{R}^{(\gamma, \delta)}}^{2} \leq c\|g\|_{\chi_{R}^{(\gamma, \delta)}}^{2} .
\end{aligned}
$$

Moreover, we use (2.38), (2.45) and the definitions of $|v|_{k, \chi_{R}^{(\alpha, \beta)}, A}, k=1,2$, to derive that

$$
\begin{aligned}
& \left\|P_{N, \alpha, \beta, \gamma, \delta}^{1} w-w\right\|_{1, \alpha, \beta, \gamma, \delta} \leq c N^{-1}|w|_{2, \chi_{R}^{(\alpha+\sigma-1, \beta+\theta-1)}, A} \\
& =c N^{-1}\left|(x+1)^{2} \partial_{x} w\right|_{1, \chi_{R}^{(\alpha+\sigma, \beta+\theta)}, A} \\
& \leq c N^{-1}\left(\left\|\partial_{x}^{2} w x^{\frac{1+\theta}{2}}(x+1)^{3-\frac{\sigma+\theta}{2}}\right\|_{\chi_{R}^{(\alpha, \beta)}}+2\left\|\partial_{x} w x^{\frac{1+\theta}{2}}(x+1)^{2-\frac{\sigma+\theta}{2}}\right\|_{\chi_{R}^{(\alpha, \beta)}}\right) \\
& \leq c N^{-1}\|g\|_{\chi_{R}^{(\gamma, \delta)} .}
\end{aligned}
$$

Finally, by taking $z=P_{N, \alpha, \beta, \gamma, \delta}^{1} v-v$ in (2.39), we use (2.38) and (2.46) to verify that

$$
\begin{aligned}
& \left|\left(P_{N, \alpha, \beta, \gamma, \delta}^{1} v-v, g\right)_{\chi_{R}^{(\gamma, \delta)}}\right|=\left|a_{\alpha, \beta, \gamma, \delta}\left(P_{N, \alpha, \beta, \gamma, \delta}^{1} v-v, P_{N, \alpha, \beta, \gamma, \delta}^{1} w-w\right)\right| \\
& \leq\left\|P_{N, \alpha, \beta, \gamma, \delta}^{1} v-v\right\|_{1, \alpha, \beta, \gamma, \delta}\left\|P_{N, \alpha, \beta, \gamma, \delta}^{1} w-w\right\|_{1, \alpha, \beta, \gamma, \delta} \\
& \leq c N^{-r}\|g\|_{\chi_{R}^{(\gamma, \delta)}}|v|_{r, \chi_{R}^{(\alpha+\sigma-1, \beta+\theta-1)}, A} .
\end{aligned}
$$

Consequently,

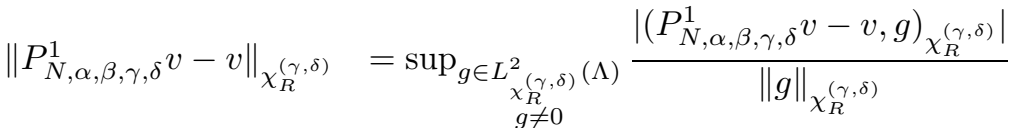

$$
\begin{aligned}
& \leq c N^{-r}|v|_{r, \chi_{R}^{(\alpha+\sigma-1, \beta+\theta-1)}, A}^{g \neq} .
\end{aligned}
$$

The result (2.37) for $0<\mu<1$ follows from (2.38), (2.47) and space interpolation.

Remark 2.2. In the Jacobi approximation, we only considered the case with $\alpha, \beta, \gamma$, $\delta>-1$. But for the Jacobi rational approximation, we introduce the parameters $\sigma$ and $\theta$ in Theorem 2.4. This enhances the flexibility and enlarges its applications. Indeed, without suitable $\sigma$ and $\theta$, we cannot derive the estimates of numerical errors of rational spectral methods for many important differential equations; see the next section. 
2.5. Orthogonal projection of functions vanishing at one extreme point. In some problems as in astrophysics and so on, the values of unknown functions are imposed at one of the extreme points, say $x=0$. In this case, we need to study another orthogonal projection. For this purpose, we let

$$
{ }_{0} H_{\alpha, \beta, \gamma, \delta}^{1}(\Lambda)=\left\{v \mid v \in H_{\alpha, \beta, \gamma, \delta}^{1}(\Lambda) \text { and } v(0)=0\right\} .
$$

The orthogonal projection ${ }_{0} P_{N, \alpha, \beta, \gamma, \delta}^{1}:{ }_{0} H_{\alpha, \beta, \gamma, \delta}^{1}(\Lambda) \rightarrow{ }_{0} \mathcal{R}_{N}$ is defined by

$$
a_{\alpha, \beta, \gamma, \delta}\left({ }_{0} P_{N, \alpha, \beta, \gamma, \delta}^{1} v-v, \phi\right)=0, \forall \phi \in{ }_{0} \mathcal{R}_{N} .
$$

Lemma 2.3. If

$$
\alpha \leq \gamma+2, \quad \beta \leq 0, \quad \gamma>-1, \quad \delta \geq 0
$$

or

$$
\alpha \leq \gamma+1, \quad \beta \leq \delta+2, \quad 0<\alpha<1, \quad \beta<1,
$$

then for any $v \in{ }_{0} H_{\chi_{R}^{(\alpha-4, \beta)}}^{1}(\Lambda)$,

$$
\|v\|_{\chi_{R}^{(\gamma, \delta)}} \leq c|v|_{1, \chi_{R}^{(\alpha-4, \beta)}}
$$

Proof. Let $u(y)=v\left(\frac{1+y}{1-y}\right)$. Clearly $u(-1)=0$. If (2.48) holds, then by Lemma 2.4 of [10], $\|u\|_{\chi^{(\gamma, \delta), I}} \leq c|u|_{1, \chi^{(\alpha, \beta), I}}$. Finally a calculation leads to the desired result. If (2.49) holds, then the same conclusion follows from Lemma 3.6 of [15] and an argument as before.

Theorem 2.5. Let $\sigma \leq 4$ and $\theta \leq 0$. If

$$
-1<\alpha+\sigma \leq \gamma+2, \quad-1<\beta+\theta \leq 0, \quad \gamma>-1, \delta \geq 0,
$$

or

$$
\alpha+\sigma \leq \gamma+1, \quad 0<\alpha+\sigma<1, \quad-1<\beta+\theta<1, \quad \gamma, \delta>-1
$$

then for any $v \in{ }_{0} H_{\alpha, \beta, \gamma, \delta}^{1}(\Lambda) \cap H_{\chi_{R}^{(\alpha+\sigma-1, \beta+\theta-1)}, A}^{r}(\Lambda), r \in \mathbb{N}$ and $r \geq 1$,

$$
\left\|{ }_{0} P_{N, \alpha, \beta, \gamma, \delta}^{1} v-v\right\|_{1, \alpha, \beta, \gamma, \delta} \leq c N^{1-r}|v|_{r, \chi_{R}^{(\alpha+\sigma-1, \beta+\theta-1)}, A} .
$$

If, in addition, (2.36) holds and $\beta>0$ or $\beta<\theta$, then for $0 \leq \mu \leq 1$,

$$
\left\|{ }_{0} P_{N, \alpha, \beta, \gamma, \delta}^{1} v-v\right\|_{\mu, \alpha, \beta, \gamma, \delta} \leq c N^{\mu-r}|v|_{r, \chi_{R}^{(\alpha+\sigma-1, \beta+\theta-1)}, A} .
$$

Proof. Let

$$
\phi(x)=\int_{0}^{x}(z+1)^{-2} P_{N-1, \alpha+\sigma, \beta+\theta}\left((z+1)^{2} \partial_{z} v(z)\right) d z \in{ }_{0} \mathcal{R}_{N} .
$$

By virtue of the projection theorem, Lemma 2.3 and Theorem 2.3, we deduce that

$$
\begin{aligned}
& \left\|{ }_{0} P_{N, \alpha, \beta, \gamma, \delta}^{1} v-v\right\|_{1, \alpha, \beta, \gamma, \delta} \leq\|\phi-v\|_{1, \alpha, \beta, \gamma, \delta} \leq c|\phi-v|_{1, \chi_{R}^{(\alpha+\sigma-4, \beta+\theta)}} \\
& =c\left\|P_{N-1, \alpha+\sigma, \beta+\theta}\left((x+1)^{2} \partial_{x} v\right)-(x+1)^{2} \partial_{x} v\right\|_{\chi_{R}^{(\alpha+\sigma, \beta+\theta)}} \\
& \leq c N^{1-r}|v|_{r, \chi_{R}^{(\alpha+\sigma-1, \beta+\theta-1)}, A} .
\end{aligned}
$$

We next prove (2.53) with condition (2.36). Let $g \in L_{\chi_{R}^{(\gamma, \delta)}}^{2}(\Lambda)$ and consider the problem

$$
a_{\alpha, \beta, \gamma, \delta}(w, z)=(g, z)_{\chi_{R}^{(\gamma, \delta)}}, \forall z \in{ }_{0} H_{\alpha, \beta, \gamma, \delta}^{1}(\Lambda)
$$


Taking $z=w$ in (2.55), we get that $\|w\|_{1, \alpha, \beta, \gamma, \delta} \leq\|g\|_{\chi_{R}^{(\gamma, \delta)}}$. It can be shown that (2.40) is still valid and $\partial_{x} w(x) \chi_{R}^{(\alpha, \beta)}(x) \rightarrow 0$, as $x \rightarrow \infty$. Moreover, (2.41) and (2.42) are also true. We can estimate $D_{1}\left(\Lambda_{2}\right)$ and $D_{2}$ in (2.42), in the same manner as in the proof of Theorem 2.4. We now estimate $D_{1}\left(\Lambda_{1}\right)$. For simplicity, let $\eta(x)=\partial_{x} w(x) \chi_{R}^{(\alpha, \beta)}(x)$. If $\beta>0$, then $\eta(0)=0$. In this case, a similar argument as in (2.44) leads to $D_{1}\left(\Lambda_{1}\right) \leq c\|g-w\|_{\chi_{R}^{(\gamma, \delta)}}^{2}$. If $\beta<\theta$, then we use (2.40) and (2.44) to obtain that

$$
\begin{aligned}
D_{1}\left(\Lambda_{1}\right) & =\int_{0}^{1} x^{-\beta+\theta-1}(x+1)^{\alpha+\beta-\sigma-\theta+8}\left(\eta(0)-\int_{0}^{x}(g(z)-w(z)) \chi_{R}^{(\gamma, \delta)}(z) d z\right)^{2} d x \\
& \leq c\|g-w\|_{\chi_{R}^{(\gamma, \delta)}}^{2}+c \eta^{2}(0) .
\end{aligned}
$$

Due to $\eta(\infty)=0$, we have from (2.40) and the Cauchy-Schwartz inequality that

$$
\eta^{2}(0) \leq\left(\int_{\Lambda}\left|\partial_{x} \eta(x)\right| d x\right)^{2}=\left(\int_{\Lambda}|g(x)-w(x)| \chi_{R}^{(\gamma, \delta)}(x) d x\right)^{2} \leq c\|g-w\|_{\chi_{R}^{(\gamma, \delta)}}^{2} .
$$

A combination of the above two estimates leads to $D_{1}\left(\Lambda_{1}\right) \leq c\|g-w\|_{\chi_{R}^{(\gamma, \delta)}}^{2}$. Finally, the desired result (2.53) follows from a duality argument and space interpolation.

2.6. Orthogonal projection of functions vanishing at both extreme points. When we study some problems in unbounded domains with homogenous boundary conditions, we have to consider another projection. For this purpose, let

$$
H_{0, \alpha, \beta, \gamma, \delta}^{1}(\Lambda)=\left\{v \mid v \in H_{\alpha, \beta, \gamma, \delta}^{1}(\Lambda) \text { and } v(0)=v(\infty)=0\right\} .
$$

The orthogonal projection $P_{N, \alpha, \beta, \gamma, \delta}^{1,0}: H_{0, \alpha, \beta, \gamma, \delta}^{1}(\Lambda) \rightarrow \mathcal{R}_{N}^{0}$ is defined by

$$
a_{\alpha, \beta, \gamma, \delta}\left(P_{N, \alpha, \beta, \gamma, \delta}^{1,0} v-v, \phi\right)=0, \forall \phi \in \mathcal{R}_{N}^{0} .
$$

Theorem 2.6. Let $\sigma \leq 4$ and $\theta \leq 0$. If

$$
-1<\alpha+\sigma, \beta+\theta<1, \quad \gamma, \delta>-1,
$$

then for $v \in H_{\chi_{R}^{(\alpha+\sigma-1, \beta+\theta-1)}, A}^{r}(\Lambda) \cap H_{0, \alpha, \beta, \gamma, \delta}^{1}(\Lambda), r \in \mathbb{N}$ and $r \geq 1$,

$$
\left\|P_{N, \alpha, \beta, \gamma, \delta}^{1,0} v-v\right\|_{1, \alpha, \beta, \gamma, \delta} \leq c N^{1-r}|v|_{r, \chi_{R}^{(\alpha+\sigma-1, \beta+\theta-1)}, A} .
$$

If, in addition, condition (2.36) holds and $\alpha>-2, \beta>0$, then for $0 \leq \mu \leq 1$,

$$
\left\|P_{N, \alpha, \beta, \gamma, \delta}^{1,0} v-v\right\|_{\mu, \alpha, \beta, \gamma, \delta} \leq c N^{\mu-r}|v|_{r, \chi_{R}^{(\alpha+\sigma-1, \beta+\theta-1)}, A} .
$$

Proof. We first assume that (2.56) holds. Let

$$
\phi^{*}(x)=\int_{0}^{x}(z+1)^{-2} P_{N-1, \alpha+\sigma, \beta+\theta}\left((z+1)^{2} \partial_{z} v(z)\right) d z, \quad \phi(x)=\phi^{*}(x)-\phi^{*}(\infty) \frac{x}{x+1} .
$$


Clearly, $\phi \in \mathcal{R}_{N}^{0}$ and $\partial_{x} \phi(x)=\partial_{x} \phi^{*}(x)-\phi^{*}(\infty)(x+1)^{-2}$. Thus by the projection theorem,

$$
\begin{aligned}
& \left\|P_{N, \alpha, \beta, \gamma, \delta}^{1,0} v-v\right\|_{1, \alpha, \beta, \gamma, \delta} \leq\|\phi-v\|_{1, \alpha, \beta, \gamma, \delta} \\
& \leq c\left\|(x+1)^{-2} P_{N-1, \alpha+\sigma, \beta+\theta}\left((x+1)^{2} \partial_{x} v\right)-(x+1)^{2} \partial_{x} v\right\|_{\chi_{R}^{(\alpha, \beta)}} \\
& +c\left(\gamma_{0}^{(\alpha+4, \beta)}\right)^{\frac{1}{2}}\left|\phi^{*}(\infty)\right|+\|\phi-v\|_{\chi_{R}^{(\gamma, \delta)}} \\
& \leq c\left\|P_{N-1, \alpha+\sigma, \beta+\theta}\left((x+1)^{2} \partial_{x} v\right)-(x+1)^{2} \partial_{x} v\right\|_{\chi_{R}^{(\alpha+\sigma, \beta+\theta)}} \\
& +c\left(\gamma_{0}^{(\alpha+4, \beta)}\right)^{\frac{1}{2}}\left|\phi^{*}(\infty)\right|+\|\phi-v\|_{\chi_{R}^{(\gamma, \delta)}} .
\end{aligned}
$$

Since $-1<\alpha+\sigma, \beta+\theta<1$, we use the Cauchy-Schwartz inequality to deduce that

$$
\begin{aligned}
& \left|\phi^{*}(\infty)\right|=\left|\int_{\Lambda}(x+1)^{-2}\left(P_{N-1, \alpha+\sigma, \beta+\theta}\left((x+1)^{2} \partial_{x} v(x)\right)-(x+1)^{2} \partial_{x} v(x)\right) d x\right| \\
& \leq\left(\gamma_{0}^{(-\alpha-\sigma,-\beta-\theta)}\right)^{\frac{1}{2}}\left\|P_{N-1, \alpha+\sigma, \beta+\theta}\left((x+1)^{2} \partial_{x} v\right)-(x+1)^{2} \partial_{x} v\right\|_{\chi_{R}^{(\alpha+\sigma, \beta+\theta)} .}
\end{aligned}
$$

On the other hand,

$$
\begin{aligned}
& \phi(x)-v(x) \\
& =\int_{0}^{x}(z+1)^{-2}\left(P_{N-1, \alpha+\sigma, \beta+\theta}\left((z+1)^{2} \partial_{z} v(z)\right)-(z+1)^{2} \partial_{z} v(z)\right) d z-\phi^{*}(\infty) \frac{x}{x+1} .
\end{aligned}
$$

Therefore, using the Cauchy-Schwartz inequality again yields that

$$
\begin{aligned}
\|\phi-v\|_{\chi_{R}^{(\gamma, \delta)}} \leq & c\left(\gamma_{0}^{(-\alpha-\sigma,-\beta-\theta)} \gamma_{0}^{(\gamma, \delta)}\right)^{\frac{1}{2}} \| P_{N-1, \alpha+\sigma, \beta+\theta}\left((x+1)^{2} \partial_{x} v\right) \\
& -(x+1)^{2} \partial_{x} v \|_{\chi_{R}^{(\alpha+\sigma, \beta+\theta)}}+c\left(\gamma_{0}^{(\gamma, \delta+2)}\right)^{\frac{1}{2}}\left|\phi^{*}(\infty)\right| .
\end{aligned}
$$

Substituting (2.60) and (2.61) into (2.59) and using Theorem 2.3 we reach result (2.57).

Next, let $\alpha>-2, \beta>0$ and let (2.36) hold. Let $g \in L_{\chi_{R}^{(\gamma, \delta)}}^{2}(\Lambda)$ and consider the auxiliary problem

$$
a_{\alpha, \beta, \gamma, \delta}(w, z)=(g, z)_{\chi_{R}^{(\gamma, \delta)}}, \forall z \in H_{0, \alpha, \beta, \gamma, \delta}^{1}(\Lambda) .
$$

Taking $z=w$ in (2.62), we get that $\|w\|_{1, \alpha, \beta, \gamma, \delta} \leq\|g\|_{\chi_{R}^{(\gamma, \delta)}}$. It can be checked that (2.40) still holds. Thanks to $\alpha>-2$ and $\beta>0$, we know that $\partial_{x} w(x) \chi_{R}^{(\alpha, \beta)}(x) \rightarrow 0$, as $x \rightarrow 0, \infty$. Moreover, (2.41) is also true. Thus, following the same line as in the derivations of (2.46) and (2.47), we reach the desired result (2.58).

\section{Applications to Differential equations of Degenerate type}

This section is for applications of the Jacobi rational spectral approximation to numerical solutions of differential equations of degenerate type on unbounded domains.

3.1. A model problem. As an example, we consider the following model problem: (3.1)

$$
\begin{cases}\partial_{t} V(x, t)+\partial_{x}\left(a(x, t) \partial_{x} V(x, t)\right)+b(x, t) \partial_{x} V(x, t) & -c(x, t) V(x, t)=F(x, t), \\ & x \in \Lambda, t \in[0, T), \\ V(x, T)=V_{0}(x), & x \in \bar{\Lambda},\end{cases}
$$


where

$$
\begin{array}{ll}
a(x, t)=a_{1}(x, t) x^{\mu_{1}}(x+1)^{\mu_{2}}, & b(x, t)=b_{1}(x, t) x^{\eta_{1}}(x+1)^{\eta_{2}} \\
c(x, t)=c_{1}(x, t) x^{\lambda_{1}}(x+1)^{\lambda_{2}}, & 0<a_{\min } \leq a_{1}(x, t) \leq a_{\max } \\
\left|b_{1}(x, t)\right| \leq b_{\max }, & 0<c_{\min } \leq c_{1}(x, t) \leq c_{\max }
\end{array}
$$

In addition, $V(x, t)$ satisfies certain asymptotic boundary condition as $x \rightarrow \infty$. The boundary condition at $x=0$ depends on the coefficients of (3.1). For instance, if $\mu_{1}>0$ and $b(0, t)<0$, then we require $V(0, t)=V_{0}(t)$, while if $\mu_{1}>0$ and $b(0, t) \geq 0$, we cannot impose any boundary condition at $x=0$.

The above problem includes many important equations in financial mathematics, such as

$\diamond$ the Black-Scholes model (see [4])

$$
\partial_{t} V(x, t)+(p-q) x \partial_{x} V(x, t)+\frac{1}{2} d^{2} x^{2} \partial_{x}^{2} V(x, t)-p V(x, t)=0,
$$

$\diamond$ the Dothan model (see [9])

$$
\partial_{t} V(x, t)+p x \partial_{x} V(x, t)+\frac{1}{2} d^{2} x^{2} \partial_{x}^{2} V(x, t)-x V(x, t)=0,
$$

$\diamond$ the Black-Derman-Toy model (see [5])

$$
\partial_{t} V(x, t)+p(t) x \partial_{x} V(x, t)+\frac{1}{2} d^{2}(t) x^{2} \partial_{x}^{2} V(x, t)-x V(x, t)=0 .
$$

If all coefficients $p, q$ and $d$ are independent of $x$, then we can derive an explicit solution of the Black-Scholes model, whereas, it is not so, even if only one of the coefficients depends on $x$. Moreover, we have no explicit solution of the corresponding multiple-dimensional model, no matter if the coefficients are independent of $x$ or not. Furthermore, we can only solve the Dothan and Black-Derman-Toy models numerically.

Next, let

$$
\begin{aligned}
& s=T-t, \quad U(x, s)=V(x, T-s), \quad U_{0}(x)=V_{0}(x), \quad f(x, s)=-F(x, T-s), \\
& \widetilde{a}(x, s)=a(x, T-s), \quad \widetilde{a}_{1}(x, s)=a_{1}(x, T-s), \quad \widetilde{b}(x, s)=b(x, T-s), \\
& \widetilde{b}_{1}(x, s)=b_{1}(x, T-s), \quad \widetilde{c}(x, s)=c(x, T-s), \quad \widetilde{c}_{1}(x, s)=c_{1}(x, T-s) .
\end{aligned}
$$

Then (3.1) is reformulated to the following convection-diffusion problem:

$$
\begin{cases}\partial_{s} U(x, s)-\partial_{x}\left(\widetilde{a}(x, s) \partial_{x} U(x, s)\right)-\widetilde{b}(x, s) \partial_{x} U(x, s) & +\widetilde{c}(x, s) U(x, s)=f(x, s), \\ & x \in \Lambda, s \in(0, T], \\ U(x, 0)=U_{0}(x), & x \in \bar{\Lambda} .\end{cases}
$$

We shall derive the weak formulation of (3.3) for two different cases and construct the spectral schemes for them.

3.2. The case $\mu_{1}>0$ and $\widetilde{b}(0, s) \geq 0$. In this case, we cannot impose any boundary condition at $x=0$. As we know, the existence and regularity of the solution of (3.3) depend not only on $\mu_{1}, \mu_{2}, \eta_{1}, \eta_{2}, \lambda_{1}$ and $\lambda_{2}$, but also on $f(x, s)$ and $U_{0}(x)$.

We introduce the spaces

$$
\begin{gathered}
\widetilde{L}_{\alpha, \beta}^{2}(\Lambda)=\left\{v \mid \int_{\Lambda} v^{2}(x) x^{\alpha}(x+1)^{\beta} d x<\infty\right\}, \\
\widetilde{H}_{\alpha, \beta, \gamma, \delta}^{1}(\Lambda)=\left\{v \mid v \in \widetilde{L}_{\gamma, \delta}^{2}(\Lambda), \partial_{x} v \in \widetilde{L}_{\alpha, \beta}^{2}(\Lambda)\right\} .
\end{gathered}
$$


Besides,

$$
(u, v)_{\alpha, \beta}=\int_{\Lambda} u(x) v(x) x^{\alpha}(x+1)^{\beta} d x, \quad\|v\|_{\alpha, \beta}=(u, v)_{\alpha, \beta}^{\frac{1}{2}} .
$$

If $U_{0}(x) \in \widetilde{L}_{\sigma_{1}, \sigma_{2}}^{2}(\Lambda)$, then the possible solution of (3.3) may belong to $L^{\infty}(0, T$; $\left.\widetilde{L}_{\sigma_{1}, \sigma_{2}}^{2}(\Lambda)\right) \cap L^{2}\left(0, T ; \widetilde{H}_{\mu_{1}+\sigma_{1}, \mu_{2}+\sigma_{2}, \sigma_{1}, \sigma_{2}}^{1}(\Lambda)\right)$. Consequently, for deriving a reasonably weak form of (3.3), we have to multiply the first equation of (3.3) by $x^{\sigma_{1}}(x+1)^{\sigma_{2}} v$ and then integrate the result over $\Lambda$. It is noted that

$$
\begin{aligned}
& -\int_{\Lambda} \partial_{x}\left(\widetilde{a}(x, s) \partial_{x} U(x, s)\right) v(x) x^{\sigma_{1}}(x+1)^{\sigma_{2}} d x \\
& =\int_{\Lambda} \widetilde{a}_{1}(x, s) \partial_{x} U(x, s) \partial_{x} v(x) x^{\mu_{1}+\sigma_{1}}(x+1)^{\mu_{2}+\sigma_{2}} d x \\
& +\int_{\Lambda} \widetilde{a}_{1}(x, s) \partial_{x} U(x, s) v(x) x^{\mu_{1}+\sigma_{1}-1}(x+1)^{\mu_{2}+\sigma_{2}-1}\left(\sigma_{1}(x+1)+\sigma_{2} x\right) d x+B(U, v)
\end{aligned}
$$

where

$$
B(U, v)=-\left.\widetilde{a}_{1}(x, s) \partial_{x} U(x, s) v(x) x^{\mu_{1}+\sigma_{1}}(x+1)^{\mu_{2}+\sigma_{2}}\right|_{0} ^{\infty} .
$$

It is easy to check that for any $v \in \widetilde{H}_{\mu_{1}+\sigma_{1}, \mu_{2}+\sigma_{2}, \sigma_{1}, \sigma_{2}}^{1}(\Lambda)$,

$$
\begin{array}{ll}
\lim _{x \rightarrow 0} x^{\frac{\sigma_{1}+1}{2}} v(x)=0, \text { a.e., } & \lim _{x \rightarrow 0} x^{\frac{\mu_{1}+\sigma_{1}+1}{2}} \partial_{x} v(x, s)=0, \text { a.e., } \\
\lim _{x \rightarrow \infty} x^{\frac{\sigma_{1}+\sigma_{2}+1}{2}} v(x)=0, \text { a.e., } & \lim _{x \rightarrow \infty} x^{\frac{\mu_{1}+\mu_{2}+\sigma_{1}+\sigma_{2}+1}{2}} \partial_{x} v(x, s)=0, \text { a.e. }
\end{array}
$$

Thus $B(U, v)=0$, a.e., for any $U, v \in \widetilde{H}_{\mu_{1}+\sigma_{1}, \mu_{2}+\sigma_{2}, \sigma_{1}, \sigma_{2}}^{1}(\Lambda)$ and $\mu_{1} \geq 2, \mu_{1}+\mu_{2} \leq$ 2. Accordingly, a weak formulation of (3.3) is to seek $U \in L^{\infty}\left(0, T ; \widetilde{L}_{\sigma_{1}, \sigma_{2}}^{2}(\Lambda)\right) \cap$ $L^{2}\left(0, T ; \widetilde{H}_{\mu_{1}+\sigma_{1}, \mu_{2}+\sigma_{2}, \sigma_{1}, \sigma_{2}}^{1}(\Lambda)\right)$ such that

$$
\begin{aligned}
\left(\partial_{s} U(s), v\right)_{\sigma_{1}, \sigma_{2}} & +\left(\widetilde{a}_{1}(s) \partial_{x} U(s), \partial_{x} v\right)_{\mu_{1}+\sigma_{1}, \mu_{2}+\sigma_{2}}+\left(\partial_{x} U(s), v\right)_{g} \\
& +\left(\widetilde{c}_{1}(s) U(s), v\right)_{\lambda_{1}+\sigma_{1}, \lambda_{2}+\sigma_{2}}=(f, v)_{\sigma_{1}, \sigma_{2}}, \\
& \forall v \in \widetilde{H}_{\mu_{1}+\sigma_{1}, \mu_{2}+\sigma_{2}, \sigma_{1}, \sigma_{2}}^{1}(\Lambda), s \in(0, T], \text { a.e. }
\end{aligned}
$$

where $(\cdot, \cdot)_{g}$ stands for the inner product with the $s$-dependent weight function $g(x, s)$,

$$
\begin{aligned}
g(x, s)= & \widetilde{a}_{1}(x, s) x^{\mu_{1}+\sigma_{1}-1}(x+1)^{\mu_{2}+\sigma_{2}-1}\left(\sigma_{1}(x+1)+\sigma_{2} x\right) \\
& -\widetilde{b}_{1}(x, s) x^{\eta_{1}+\sigma_{1}}(x+1)^{\eta_{2}+\sigma_{2}} .
\end{aligned}
$$

It is easy to see that if

$$
2 \leq \mu_{1} \leq 2 \eta_{1}, \quad 2\left(\eta_{1}+\eta_{2}\right) \leq \mu_{1}+\mu_{2} \leq 2,
$$

then

$$
\left|\left(\partial_{x} U(s), v\right)_{g}\right| \leq c|| \partial_{x} U\left\|_{\mu_{1}+\sigma_{1}, \mu_{2}+\sigma_{2}}|| v\right\|_{\sigma_{1}, \sigma_{2}} .
$$

If, in addition, $f \in L^{2}\left(0, T ;\left(\widetilde{H}_{\mu_{1}+\sigma_{1}, \mu_{2}+\sigma_{2}, \sigma_{1}, \sigma_{2}}^{1}(\Lambda)\right)^{\prime}\right)$ and $U_{0} \in \widetilde{L}_{\sigma_{1}, \sigma_{2}}^{2}(\Lambda)$, then (3.4) has a unique solution. In particular, if $\widetilde{b}_{1}(x, s) \equiv 0$, then the corresponding conditions can be weakened as $\mu_{1} \geq 2$ and $\mu_{1}+\mu_{2} \leq 2$.

Let $P_{N,-\sigma_{1}-\sigma_{2}-2, \sigma_{1}}$ be the orthogonal projection given by (2.18), and let $u_{N}(s)$ be the approximation to $U(s)$. The Jacobi rational spectral scheme for (3.4) is to 
find $u_{N}(s) \in \mathcal{R}_{N}$ such that

$$
\left\{\begin{array}{l}
\left(\partial_{s} u_{N}(s), \phi\right)_{\sigma_{1}, \sigma_{2}}+\left(\widetilde{a}_{1}(s) \partial_{x} u_{N}(s), \partial_{x} \phi\right)_{\mu_{1}+\sigma_{1}, \mu_{2}+\sigma_{2}}+\left(\partial_{x} u_{N}(s), \phi\right)_{g} \\
+\left(\widetilde{c}_{1}(s) u_{N}(s), \phi\right)_{\lambda_{1}+\sigma_{1}, \lambda_{2}+\sigma_{2}}=(f, \phi)_{\sigma_{1}, \sigma_{2}}, \quad \forall \phi \in \mathcal{R}_{N}, s \in(0, T], \\
u_{N}(0)=P_{N,-\sigma_{1}-\sigma_{2}-2, \sigma_{1}} U_{0} .
\end{array}\right.
$$

We now deal with the convergence of (3.6). To do this, we need a specific orthogonal projection $\Pi_{N}^{1}: \widetilde{H}_{\mu_{1}+\sigma_{1}, \mu_{2}+\sigma_{2}, \sigma_{1}, \sigma_{2}}^{1}(\Lambda) \cap \widetilde{L}_{\lambda_{1}+\sigma_{1}, \lambda_{2}+\sigma_{2}}^{2}(\Lambda) \rightarrow \mathcal{R}_{N}$, defined by

$$
\begin{aligned}
\left(\partial_{x}\left(\Pi_{N}^{1} v-v\right), \partial_{x} \phi\right)_{\mu_{1}+\sigma_{1}, \mu_{2}+\sigma_{2}} & +\left(\Pi_{N}^{1} v-v, \phi\right)_{\lambda_{1}+\sigma_{1}, \lambda_{2}+\sigma_{2}} \\
& +\left(\Pi_{N}^{1} v-v, \phi\right)_{\sigma_{1}, \sigma_{2}}=0, \quad \forall \phi \in \mathcal{R}_{N} .
\end{aligned}
$$

For a description of approximation errors, we use the following notation:

$$
\begin{gathered}
B_{\mu_{1}, \mu_{2}, \sigma_{1}, \sigma_{2}, \sigma, \theta}^{r}(\Lambda)=H_{\chi_{R}^{\left(-\mu_{1}-\mu_{2}-\sigma_{1}-\sigma_{2}+\sigma-3, \mu_{1}+\sigma_{1}+\theta-1\right)}, A}^{r}(\Lambda), \\
|v|_{B_{\mu_{1}, \mu_{2}, \sigma_{1}, \sigma_{2}, \sigma, \theta}^{r}}^{r}=|v|_{r, \chi_{R}^{\left(-\mu_{1}-\mu_{2}-\sigma_{1}-\sigma_{2}+\sigma-3, \mu_{1}+\sigma_{1}+\theta-1\right)}, A} .
\end{gathered}
$$

Following the same line of reasoning as in the proof of Theorem 2.4, we can prove the following result; see the Appendix.

Lemma 3.1. Let

$$
\begin{gathered}
\sigma=\min \left(\mu_{1}+\mu_{2}+2, \mu_{1}+\mu_{2}-\lambda_{1}-\lambda_{2}+2,4\right), \\
\theta=\min \left(-\mu_{1}+2,-\mu_{1}+\lambda_{1}+2,0\right) .
\end{gathered}
$$

If

$$
\sigma_{1}+\sigma_{2}<\min \left(-\lambda_{1}-\lambda_{2}-1,-1\right), \quad \sigma_{1}>\max \left(-\lambda_{1}-1,-1\right),
$$

then for any $v \in B_{\mu_{1}, \mu_{2}, \sigma_{1}, \sigma_{2}, \sigma, \theta}^{r}(\Lambda), r \in \mathbb{N}$ and $r \geq 1$,

$$
\begin{aligned}
\left\|\partial_{x}\left(\Pi_{N}^{1} v-v\right)\right\|_{\mu_{1}+\sigma_{1}, \mu_{2}+\sigma_{2}} & +\left\|\Pi_{N}^{1} v-v\right\|_{\lambda_{1}+\sigma_{1}, \lambda_{2}+\sigma_{2}}+\left\|\Pi_{N}^{1} v-v\right\|_{\sigma_{1}, \sigma_{2}} \\
& \leq c N^{1-r}|v|_{B_{\mu_{1}, \mu_{2}, \sigma_{1}, \sigma_{2}, \sigma, \theta}^{r}} .
\end{aligned}
$$

We are now in a position to estimate the numerical error. Let $U_{N}=\Pi_{N}^{1} U$. Then by (3.4),

$$
\begin{aligned}
& \left(\partial_{s} U_{N}(s), \phi\right)_{\sigma_{1}, \sigma_{2}}+\left(\widetilde{a}_{1}(s) \partial_{x} U_{N}(s), \partial_{x} \phi\right)_{\mu_{1}+\sigma_{1}, \mu_{2}+\sigma_{2}}+\left(\partial_{x} U_{N}(s), \phi\right)_{g} \\
& +\left(\widetilde{c}_{1}(s) U_{N}(s), \phi\right)_{\lambda_{1}+\sigma_{1}, \lambda_{2}+\sigma_{2}}+\sum_{j=1}^{4} G_{j}(\phi, s)=(f, \phi)_{\sigma_{1}, \sigma_{2}}, \quad \forall \phi \in \mathcal{R}_{N}, s \in(0, T]
\end{aligned}
$$

where

$$
\begin{aligned}
& G_{1}(\phi, s)=\left(\partial_{s} U(s)-\partial_{s} U_{N}(s), \phi\right)_{\sigma_{1}, \sigma_{2}}, \\
& G_{2}(\phi, s)=\left(\widetilde{a}_{1}(s)\left(\partial_{x} U(s)-\partial_{x} U_{N}(s)\right), \partial_{x} \phi\right)_{\mu_{1}+\sigma_{1}, \mu_{2}+\sigma_{2}}, \\
& G_{3}(\phi, s)=\left(\partial_{x} U(s)-\partial_{x} U_{N}(s), \phi\right)_{g}, \\
& G_{4}(\phi, s)=\left(\widetilde{c}_{1}(s)\left(U(s)-U_{N}(s)\right), \phi\right)_{\lambda_{1}+\sigma_{1}, \lambda_{2}+\sigma_{2}} .
\end{aligned}
$$


Further, let $\widetilde{U}_{N}=u_{N}-U_{N}$. Subtracting (3.10) from (3.6) gives that

$$
\left\{\begin{array}{l}
\left(\partial_{s} \widetilde{U}_{N}(s), \phi\right)_{\sigma_{1}, \sigma_{2}}+\left(\widetilde{a}_{1}(s) \partial_{x} \widetilde{U}_{N}(s), \partial_{x} \phi\right)_{\mu_{1}+\sigma_{1}, \mu_{2}+\sigma_{2}}+\left(\partial_{x} \widetilde{U}_{N}(s), \phi\right)_{g} \\
+\left(\widetilde{c}_{1}(s) \widetilde{U}_{N}(s), \phi\right)_{\lambda_{1}+\sigma_{1}, \lambda_{2}+\sigma_{2}}=\sum_{j=1}^{4} G_{j}(\phi, s), \quad \forall \phi \in \mathcal{R}_{N}, s \in(0, T] \\
\widetilde{U}_{N}(0)=P_{N,-\sigma_{1}-\sigma_{2}-2, \sigma_{1}} U_{0}-\Pi_{N}^{1} U_{0}
\end{array}\right.
$$

By taking $\phi=2 \widetilde{U}_{N}(s)$ in (3.11), we obtain that

$$
\begin{aligned}
& \partial_{s}\left\|\widetilde{U}_{N}(s)\right\|_{\sigma_{1}, \sigma_{2}}^{2}+2 a_{m i n}\left\|\partial_{x} \widetilde{U}_{N}(s)\right\|_{\mu_{1}+\sigma_{1}, \mu_{2}+\sigma_{2}}^{2}+2 c_{m i n}\left\|\widetilde{U}_{N}(s)\right\|_{\lambda_{1}+\sigma_{1}, \lambda_{2}+\sigma_{2}}^{2} \\
& \leq 2 \sum_{j=1}^{5}\left|G_{j}\left(\widetilde{U}_{N}, s\right)\right|
\end{aligned}
$$

where $G_{5}\left(\widetilde{U}_{N}, s\right)=-\left(\partial_{x} \widetilde{U}_{N}(s), \widetilde{U}_{N}(s)\right)_{g}$. Therefore it remains to estimate the terms $\left|G_{j}\left(\widetilde{U}_{N}, s\right)\right|$.

Let $\sigma$ and $\theta$ be the same as in (3.7). If condition (3.8) holds, then by virtue of (3.9),

$$
\begin{aligned}
2\left|G_{1}\left(\widetilde{U}_{N}, s\right)\right| & \leq\left\|\widetilde{U}_{N}(s)\right\|_{\sigma_{1}, \sigma_{2}}^{2}+c\left\|\partial_{s} U(s)-\partial_{s} U_{N}(s)\right\|_{\sigma_{1}, \sigma_{2}}^{2} \\
& \leq\left\|\widetilde{U}_{N}(s)\right\|_{\sigma_{1}, \sigma_{2}}^{2}+c N^{2-2 r}\left|\partial_{s} U(s)\right|_{B_{\mu_{1}, \mu_{2}, \sigma_{1}, \sigma_{2}, \sigma, \theta}^{2}}^{2}
\end{aligned} .
$$

For the same reason, we have that

$$
\begin{aligned}
& 2\left|G_{2}\left(\widetilde{U}_{N}, s\right)\right| \leq \frac{1}{2} a_{m i n}\left\|\partial_{x} \widetilde{U}_{N}(s)\right\|_{\mu_{1}+\sigma_{1}, \mu_{2}+\sigma_{2}}^{2}+c N^{2-2 r}|U(s)|_{B_{\mu_{1}, \mu_{2}, \sigma_{1}, \sigma_{2}, \sigma, \theta}^{r}}^{2} \\
& \quad 2\left|G_{4}\left(\widetilde{U}_{N}, s\right)\right| \leq c_{m i n}\left\|\widetilde{U}_{N}(s)\right\|_{\lambda_{1}+\sigma_{1}, \lambda_{2}+\sigma_{2}}^{2}+c N^{2-2 r}|U(s)|_{B_{\mu_{1}, \mu_{2}, \sigma_{1}, \sigma_{2}, \sigma, \theta}^{r}}^{2}
\end{aligned}
$$

If, in addition, condition (3.5) holds, then we use (3.9) to deduce that

$$
\begin{aligned}
2\left|G_{3}\left(\widetilde{U}_{N}, s\right)\right| & \leq\left\|\widetilde{U}_{N}(s)\right\|_{\sigma_{1}, \sigma_{2}}^{2}+c\left\|\partial_{x}\left(U(s)-U_{N}(s)\right)\right\|_{\mu_{1}+\sigma_{1}, \mu_{2}+\sigma_{2}}^{2} \\
& \leq\left\|\widetilde{U}_{N}(s)\right\|_{\sigma_{1}, \sigma_{2}}^{2}+c N^{2-2 r}|U(s)|_{B_{\mu_{1}, \mu_{2}, \sigma_{1}, \sigma_{2}, \sigma, \theta}^{2}}^{2} \cdot
\end{aligned}
$$

Similarly,

$$
2\left|G_{5}\left(\widetilde{U}_{N}, s\right)\right| \leq c\left\|\widetilde{U}_{N}(s)\right\|_{\sigma_{1}, \sigma_{2}}^{2}+\frac{1}{2} a_{m i n}\left\|\partial_{x} \widetilde{U}_{N}(s)\right\|_{\mu_{1}+\sigma_{1}, \mu_{2}+\sigma_{2}}^{2} .
$$

Besides, by Theorem 2.3 and Lemma 3.1,

$$
\begin{aligned}
\left\|\widetilde{U}_{N}(0)\right\|_{\sigma_{1}, \sigma_{2}}^{2} & \leq\left\|U_{0}-P_{N,-\sigma_{1}-\sigma_{2}-2, \sigma_{1}} U_{0}\right\|_{\sigma_{1}, \sigma_{2}}^{2}+\left\|U_{0}-\Pi_{N}^{1} U_{0}\right\|_{\sigma_{1}, \sigma_{2}}^{2} \\
& \leq c N^{2-2 r}\left|U_{0}\right|_{r-1, \chi_{R}^{\left(-\sigma_{1}-\sigma_{2}-2, \sigma_{1}\right)}, A}^{2}+c N^{2-2 r}\left|U_{0}\right|_{B_{\mu_{1}, \mu_{2}, \sigma_{1}, \sigma_{2}, \sigma, \theta}^{r}}^{2} .
\end{aligned}
$$

Now, let

$E(v, s)=\|v(s)\|_{\sigma_{1}, \sigma_{2}}^{2}+\int_{0}^{s}\left(a_{m i n}\left\|\partial_{x} v(\xi)\right\|_{\mu_{1}+\sigma_{1}, \mu_{2}+\sigma_{2}}^{2}+c_{m i n}\|v(\xi)\|_{\lambda_{1}+\sigma_{1}, \lambda_{2}+\sigma_{2}}^{2}\right) d \xi$.

Substituting (3.13)-(3.18) into (3.12) and integrating the result with respect to $s$, we obtain

$$
E\left(\widetilde{U}_{N}, s\right) \leq c \int_{0}^{s} E\left(\widetilde{U}_{N}, \xi\right) d \xi+\rho(U, s)
$$


where

$$
\begin{aligned}
\rho(U, s)=c N^{2-2 r} & \left(\int_{0}^{s}\left(|U(\xi)|_{B_{\mu_{1}, \mu_{2}, \sigma_{1}, \sigma_{2}, \sigma, \theta}^{r}}^{2}+\left|\partial_{\xi} U(\xi)\right|_{B_{\mu_{1}, \mu_{2}, \sigma_{1}, \sigma_{2}, \sigma, \theta}^{r}}^{2}\right) d \xi\right. \\
& \left.+\left|U_{0}\right|_{r-1, \chi_{R}^{\left(-\sigma_{1}-\sigma_{2}-2, \sigma_{1}\right), A}}^{2}+\left|U_{0}\right|_{B_{\mu_{1}, \mu_{2}, \sigma_{1}, \sigma_{2}, \sigma, \theta}^{r}}^{2}\right) .
\end{aligned}
$$

Finally, applying the Gronwall inequality to (3.19), we arrive at $E\left(\widetilde{U}_{N}, s\right) \leq$ $e^{c s} \rho(U, s)$. This with (3.9) leads to the following conclusion.

Theorem 3.1. Let $\sigma$ and $\theta$ be the same as in (3.7), and assume conditions (3.5) and (3.8) hold. If for integer $r \geq 1, U \in H^{1}\left(0, T ; B_{\mu_{1}, \mu_{2}, \sigma_{1}, \sigma_{2}, \sigma, \theta}^{r}(\Lambda)\right)$ and $U_{0} \in$ $H_{\chi_{R}^{\left(-\sigma_{1}-\sigma_{2}-2, \sigma_{1}\right)}, A}^{r-1}(\Lambda) \cap B_{\mu_{1}, \mu_{2}, \sigma_{1}, \sigma_{2}, \sigma, \theta}^{r}(\Lambda)$, then for all $s \in[0, T]$,

$$
E\left(U-u_{N}, s\right) \leq b^{*} N^{2-2 r}
$$

where the constant $b^{*}>0$ depends only on the semi-norms of $U$ and $U_{0}$ in the mentioned spaces. Moreover, if $\widetilde{b}_{1}(x, s) \equiv 0$, then condition (3.5) can be weakened as $\mu_{1} \geq 2$ and $\mu_{1}+\mu_{2} \leq 2$.

3.3. The case $\mu_{1}>0$ and $\widetilde{b}(0, s)<0$. In this case, we should impose a boundary condition at $x=0$, say, $U(0, s)=0$. Let

$$
{ }_{0} \widetilde{H}_{\alpha, \beta, \gamma, \delta}^{1}(\Lambda)=\left\{v \mid v(0)=0, v \in \widetilde{H}_{\alpha, \beta, \gamma, \delta}^{1}(\Lambda)\right\} .
$$

We focus on the case $U_{0}(x) \in \widetilde{L}_{\sigma_{1}, \sigma_{2}}^{2}(\Lambda)$. Then the possible solution $U(x, t)$ might belong to $L^{\infty}\left(0, T ; \widetilde{L}_{\sigma_{1}, \sigma_{2}}^{2}(\Lambda)\right) \cap L^{2}\left(0, T ; \tilde{H}_{\mu_{1}+\sigma_{1}, \mu_{2}+\sigma_{2}, \sigma_{1}, \sigma_{2}}^{1}(\Lambda)\right)$. We can derive the same weak formulation and Jacobi rational spectral scheme as in (3.4) and (3.6), but the spaces $\widetilde{H}_{\mu_{1}+\sigma_{1}, \mu_{2}+\sigma_{2}, \sigma_{1}, \sigma_{2}}^{1}(\Lambda)$ and $\mathcal{R}_{N}$ are now replaced by ${ }_{0} \widetilde{H}_{\mu_{1}+\sigma_{1}, \mu_{2}+\sigma_{2}, \sigma_{1}, \sigma_{2}}^{1}(\Lambda)$ and ${ }_{0} \mathcal{R}_{N}$, respectively.

We now turn to convergence. For this purpose, we introduce a specific orthogonal projection ${ }_{0} \Pi_{N}^{1}:{ }_{0} \widetilde{H}_{\mu_{1}+\sigma_{1}, \mu_{2}+\sigma_{2}, \sigma_{1}, \sigma_{2}}^{1}(\Lambda) \cap \widetilde{L}_{\lambda_{1}+\sigma_{1}, \lambda_{2}+\sigma_{2}}^{2}(\Lambda) \rightarrow_{0} \mathcal{R}_{N}$, defined by

$$
\begin{aligned}
& \left(\partial_{x}\left({ }_{0} \Pi_{N}^{1} v-v\right), \partial_{x} \phi\right)_{\mu_{1}+\sigma_{1}, \mu_{2}+\sigma_{2}}+\left({ }_{0} \Pi_{N}^{1} v-v, \phi\right)_{\lambda_{1}+\sigma_{1}, \lambda_{2}+\sigma_{2}}+\left({ }_{0} \Pi_{N}^{1} v-v, \phi\right)_{\sigma_{1}, \sigma_{2}} \\
& \quad=0, \quad \forall \phi \in \mathcal{R}_{0} \mathcal{R}_{N} .
\end{aligned}
$$

Let

$$
\sigma=\min \left(\mu_{1}+\mu_{2}+2, \mu_{1}+\mu_{2}-\lambda_{1}-\lambda_{2}+2,4\right), \quad \theta=-\mu_{1}-\sigma_{1} .
$$

We have the approximation result stated below, which can be proved by Lemma 2.3 with (2.48) and the same argument as for the proof of Lemma 3.1.

Lemma 3.2. If

$$
\sigma_{1}+\sigma_{2}<\min \left(-\lambda_{1}-\lambda_{2}-1,-1\right), \quad \sigma_{1} \geq \max \left(-\lambda_{1}, 0\right),
$$

then for any $v \in \in_{0} \widetilde{H}_{\mu_{1}+\sigma_{1}, \mu_{2}+\sigma_{2}, \sigma_{1}, \sigma_{2}}^{1}(\Lambda) \cap B_{\mu_{1}, \mu_{2}, \sigma_{1}, \sigma_{2}, \sigma, \theta}^{r}(\Lambda), r \in \mathbb{N}$ and $r \geq 1$,

$$
\begin{gathered}
\left\|\partial_{x}\left({ }_{0} \Pi_{N}^{1} v-v\right)\right\|_{\mu_{1}+\sigma_{1}, \mu_{2}+\sigma_{2}} \\
+\left\|_{0} \Pi_{N}^{1} v-v\right\|_{\lambda_{1}+\sigma_{1}, \lambda_{2}+\sigma_{2}}+\|\|_{0} \Pi_{N}^{1} v-v \|_{\sigma_{1}, \sigma_{2}} \\
\leq c N^{1-r}|v|_{B_{\mu_{1}, \mu_{2}, \sigma_{1}, \sigma_{2}, \sigma, \theta}^{r}} .
\end{gathered}
$$

Let $U_{N}={ }_{0} \Pi_{N}^{1} U$. Then we follow the same line of reasoning as in the proof of Theorem 3.1 to obtain the following result on convergence. 
Theorem 3.2. Let $\sigma$ and $\theta$ be the same as in (3.20), and let conditions (3.5) and (3.21) hold. If for integer $r \geq 1, U \in H^{1}\left(0, T ;_{0} \widetilde{H}_{\mu_{1}+\sigma_{1}, \mu_{2}+\sigma_{2}, \sigma_{1}, \sigma_{2}}^{1}(\Lambda) \cap\right.$ $\left.B_{\mu_{1}, \mu_{2}, \sigma_{1}, \sigma_{2}, \sigma, \theta}^{r}(\Lambda)\right), U_{0} \in H_{\chi_{R}^{\left(-\sigma_{1}-\sigma_{2}-2, \sigma_{1}\right)}, A}^{r-1}(\Lambda) \cap B_{\mu_{1}, \mu_{2}, \sigma_{1}, \sigma_{2}, \sigma, \theta}^{r}(\Lambda)$, then for $s \in$ $[0, T]$,

$$
E\left(U-u_{N}, s\right) \leq b^{* *} N^{2-2 r}
$$

where the constant $b^{* *}>0$ depends only on the semi-norms of $U$ and $U_{0}$ in the mentioned spaces.

Using Lemma 2.3 with (2.49), we can obtain results similar to Lemma 3.2 and Theorem 3.2.

Remark 3.1. By space interpolation, the results of Theorems 3.1 and 3.2 are also valid for any $r \geq 1$. But $b^{*}$ and $b^{* *}$ now depend on the norms of $U$ and $U_{0}$ in the corresponding spaces.

3.4. Other applications. We can use the Jacobi rational approximation for a lot of other problems. For example, we consider the Cox-Ingersoll-Ross model (see [8])

$$
\partial_{s} V(\rho, s)+(b-a \rho) \rho \partial_{\rho} V(\rho, s)+\frac{1}{2} d^{2} \rho \partial_{\rho}^{2} V(\rho, s)-\rho V(\rho, s)=0 .
$$

We design the Jacobi rational spectral method for this equation in the same manner as in the last two subsections. For numerical analysis, we need different orthogonal projections depending on the sign of $d^{2}-2 b$.

We can use the mixed Jacobi rational-Legendre spectral method or the combined Jacobi rational spectral-finite difference method, with domain decomposition, to solve the two-dimensional model with jump coefficient:

$$
\begin{aligned}
\partial_{t} v(x, y, t)+ & H(x) \partial_{y} v(x, y, t)+\left(\frac{1}{2} \sigma^{2}+q-r\right) \partial_{x} v(x, y, t)-\frac{1}{2} \sigma^{2} \partial_{x}^{2} v(x, y, t) \\
& +r v(x, y, t)=0, \quad-\infty<x<\infty, 0<y, t<T
\end{aligned}
$$

where $H(x)$ is the Heaviside function.

An open and interesting problem is how to generalize this method to American option models, which is related to moving boundary and variational inequalities.

Our method is also applicable to singular problems and differential equations in spherical geometry, for instance, the three-dimensional Klein-Gordon equation:

$$
\begin{aligned}
& \partial_{t}^{2} U(\rho, \lambda, \theta, t)-\frac{1}{\rho^{2}} \partial_{\rho}\left(\rho^{2} \partial_{\rho} U(\rho, \lambda, \theta, t)\right)-\frac{1}{\rho^{2} \cos \theta} \partial_{\theta}\left(\cos \theta \partial_{\theta} U(\rho, \lambda, \theta, t)\right) \\
& -\frac{1}{\rho^{2} \cos ^{2} \theta} \partial_{\lambda}^{2} U(\rho, \lambda, \theta, t)+\gamma U(t, \rho, \lambda, \theta)+U^{3}(\rho, \lambda, \theta, t)=f(\rho, \lambda, \theta, t), \quad \gamma=-1,1 .
\end{aligned}
$$

Since the longitude $\lambda$ and the latitude $\theta$ vary in finite intervals, there exists only the variable $\rho$ varying from 0 to $\infty$. Moreover, we can use spherical harmonic approximation and benefit from its orthogonality. This simplifies the computation. The remaining problem is how to approximate the above equation in the radial direction. Surely, we can use the method in this paper.

Another important application is numerical simulation of fluid flow in an infinite strip; see [24]. Similarly, we can solve the exterior problem of fluid flow and some problems in astrophysics by using the proposed method coupled with other techniques. 


\section{NumericAl RESUlts}

In this section, we present some numerical results. We consider the Dothantype model for which we cannot derive an explicit expression of an exact solution. This problem corresponds to (3.1) with $a(x, t)=\frac{1}{2} d^{2} x^{2}, b(x, t)=\left(p-d^{2}\right) x$ and $c(x, t)=x$. Thereby, we have that $\mu_{1}=2, \mu_{2}=\eta_{2}=\lambda_{2}=0$ and $\eta_{1}=\lambda_{1}=1$ in (3.2). Moreover, $\sigma=3$ and $\theta=0$ in (3.7).

We shall use scheme (3.6) to solve the above problem, with suitable $\sigma_{1}$ and $\sigma_{2}$ depending on the singularity of the initial state $U(x, 0)$. In actual computation, we take $d=0.03, p=0.05, T=1$, and the base functions $R_{l}^{\left(-\sigma_{2}-2,0\right)}(x), 0 \leq l \leq N$. We use the Crank-Nicolson difference discretization in time, with the step size $\tau=10^{-3}$.

We focus on call-option solutions which grow to infinity as $x$ tends to infinity. It is difficult to use the finite difference method to simulate them directly. We take the test function $U(x, s)=\frac{1}{2}(x+1)^{h}+(\sin s x-2) e^{-x}+3, h>0$. Obviously, $U(x, 0) \in \widetilde{L}_{0, \sigma_{2}}^{2}(\Lambda)$ for any $\sigma_{2}<-2 h-1$. It can be checked that for any $r<-\sigma_{2}-$ $2 h-1, U \in L^{\infty}\left(0, T ; B_{2,0,0, \sigma_{2}, 3,0}^{r}(\Lambda)\right)$. According to Remark 3.1, if $\sigma_{2}<-2 h-2$, then the scheme (3.6) is convergent.

We first take $h=1$ and $\sigma_{2}=-3.1,-4.1,-5.1,-6.1$. In Figure 1, we plot the global weighted errors $\log _{10}\left\|U(1)-u_{N}(1)\right\|_{0, \sigma_{2}}$ vs. $\log _{10} N$, which indicate the convergence of scheme (3.6) with $\sigma_{2}<-4$ and so coincide well with theoretical analysis. Furthermore, according to Remark 3.1, for sufficiently small $\tau$, the global weighted errors do not exceed $b^{*} N^{\sigma_{2}+4}$. But we find from Figure 1 that for $\sigma_{2}=-4.1,-5.1,-6.1$, the global weighted errors are of the order $N^{-1.7}, N^{-2.4}$ and $N^{-3.2}$, respectively, which are much smaller than the predicted ones. Moreover, it is shown that scheme (3.6) with $\sigma_{2}=-3.1$ is also convergent. Therefore our method provides better numerical results than the predicted ones. In Figure 2, we plot the pointwise absolute errors $\left|U(x, 1)-u_{N}(x, 1)\right|$ with $N=64$, which are of the order $10^{-3}$. They demonstrate that scheme (3.6) provides accurate numerical results even for call-option solutions growing rapidly as $x \rightarrow \infty$. In Figure 3, we plot the pointwise relative errors $\left|\frac{U(x, 1)-u_{N}(x, 1)}{U(x, 1)}\right|$ with $N=64$. For $\sigma_{2}=-3.1,-4.1,-5.1,-6.1$, they are of the order $10^{-4}, 10^{-5}, 10^{-5}$ and $10^{-5}$, respectively. They show again the high accuracy of scheme (3.6).

As predicted by Remark 3.1, the convergence rate of scheme (3.6) depends on the regularity of the exact solution. We now take $h=\frac{1}{2}$. In this case, scheme (3.6) is convergent for $\sigma_{2}<-3$. We plot the global weighted errors in Figure 4 . For $\sigma_{2}=-3.1,-4.1,-5.1,-6.1$, they are of the order $N^{-1.8}, N^{-2.8}, N^{-3.4}$ and $N^{-3.7}$, respectively. Clearly, they are smaller than the predicted ones which are of the order $N^{\sigma_{2}+3}$. The global weighted errors with $h=\frac{1}{2}$ are also smaller than those with $h=1$. This implies that the more regular the solution, the more accurate the numerical results. The above facts coincide very well with theoretical analysis. In Figure 5, we plot the pointwise absolute errors with $N=64$. For $\sigma_{2}=-3.1,-4.1,-5.1,-6.1$, they are of the order $10^{-4}, 10^{-5}, 10^{-5}$ and $10^{-5}$, respectively. In Figure 6 , we plot the pointwise relative errors with $N=64$, which are of the order $10^{-6}$. These results show again the high accuracy of scheme (3.6). 


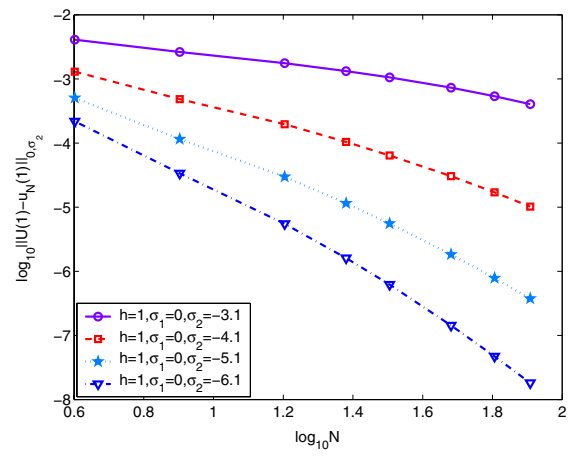

Figure 1. Global weighted errors.

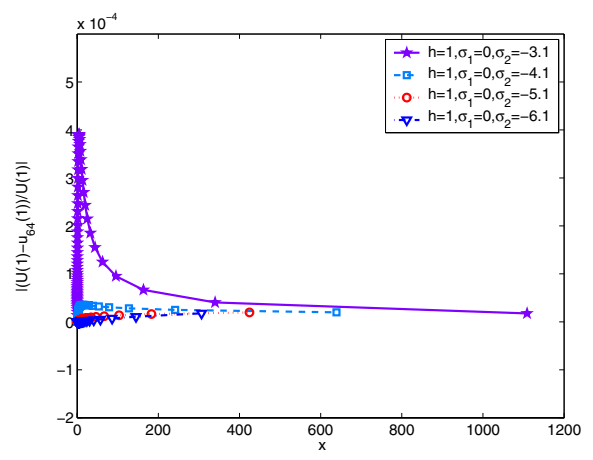

Figure 3. Pointwise relative errors.

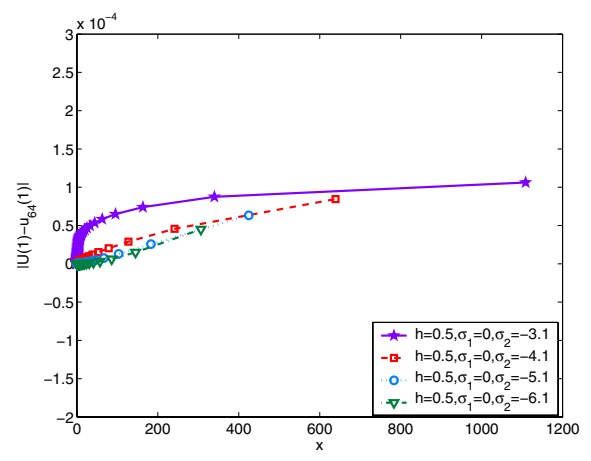

Figure 5. Pointwise absolute errors.

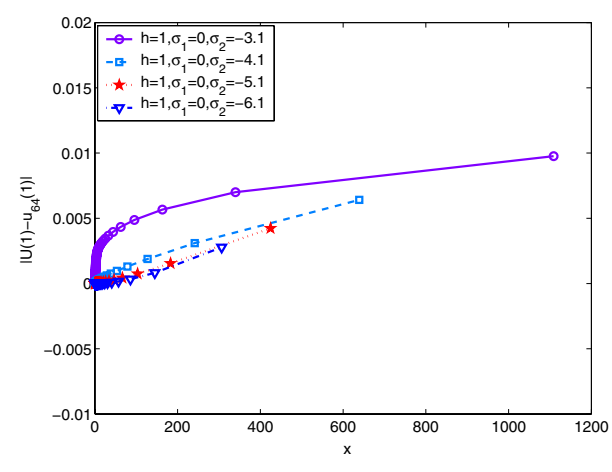

Figure 2. Pointwise absolute errors.

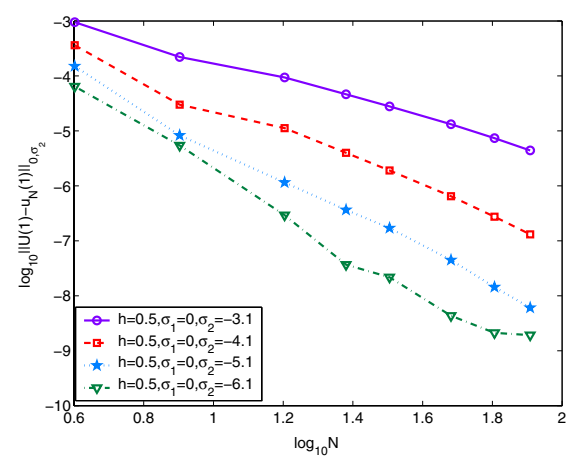

FIGURE 4. Global weighted errors.

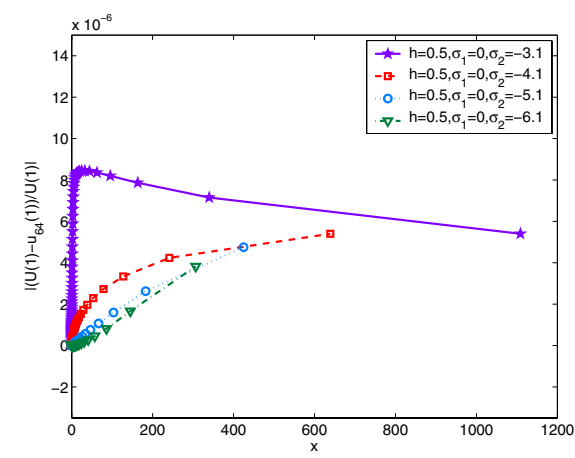

Figure 6. Pointwise relative errors.

\section{CONCLUding REMARKS}

In the existing rational spectral methods, the basis functions are induced only by Legendre or Chebyshev polynomials, which are mutually orthogonal with fixed weight functions and may not be the most appropriate for underlying problems. In 
this paper, we introduced a new orthogonal system of rational functions induced by general Jacobi polynomials with the parameters $\alpha$ and $\beta$. It is more flexible in applications. In particular, we could regulate $\alpha$ and $\beta$, so that the systems are mutually orthogonal in certain weighted Sobolev spaces to which the exact solutions of underlying problems belong. This enlarges its applications essentially.

We usually considered orthogonal projections in the standard weighted Sobolev spaces. However, in many practical problems, the coefficients of terms involving derivatives of unknown functions of different orders degenerate or grow up in different ways. This fact limits the applications of the rational spectral method seriously. In this paper, we investigated orthogonal projections in nonuniformly weighted Sobolev spaces, which form the mathematical foundation of spectral methods for a large class of differential equations with the coefficients degenerating or growing up at certain points.

In the previous work, one analyzed the rational spectral approximation in a twisted way, and so the results are not optimal. We provided a completely new and powerful framework for the Jacobi rational approximation, which leads to optimal error estimates.

As an example of applications, we proposed the Jacobi rational spectral method for the model problem (3.1) which appears frequently in financial mathematics and other fields. The numerical results indicated the efficiency of this new approach. The techniques used in this paper are also applicable to many other problems as discussed in the last part of section 3 .

We can use the rational basis functions $R_{l}(x)=J_{l}^{(\alpha, \beta)}\left(x\left(x^{2}+1\right)^{-\frac{1}{2}}\right)$ to design rational spectral methods for various problems on the whole line, such as numerical simulations of heteroclinic solutions of Fisher and Nagumo equations in biology.

Recently, some authors considered the irrational spectral method with the basis functions $I_{l}^{(\alpha, \gamma, \delta)}(x)=(x+1)^{-\gamma} J_{l}^{(\alpha, 0)}\left(1-2(x+1)^{-\delta}\right), \delta>0, x>0$ (cf. [12]). By a suitable choice of parameters $\alpha, \gamma$ and $\delta$, they form an orthogonal system with certain proper weight and fit well the asymptotic behavior of the solution of the underlying problem. But the corresponding spectral method is available only for problems with the coefficient degenerating at infinity. Conversely, in this work, we may adjust two parameters $\alpha$ and $\beta$ involved in the rational functions $R_{l}^{(\alpha, \beta)}(x)$. Thus the related spectral method is also useful for problems with singularities at $x=0, \infty$. This fact enlarges its applications. On the other hand, we may follow the same lines of reasoning as in this paper to analyze directly the irrational spectral method proposed in 12 and derive better results. Furthermore, we could combine this work with the idea of [12] to design a new irrational Jacobi spectral method by using the basis functions $I_{l}^{(\alpha, \beta, \gamma, \lambda, \delta)}(x)=x^{\lambda}(x+1)^{-\gamma} J_{l}^{(\alpha, \beta)}\left(1-2(x+1)^{-\delta}\right)$, which might provide better numerical results sometimes.

\section{APPENDix}

We prove Lemma 3.1. Let $\alpha=-\mu_{1}-\mu_{2}-\sigma_{1}-\sigma_{2}-2, \beta=\mu_{1}+\sigma_{1}$, and

$$
\phi(x)=\int_{0}^{x}(z+1)^{-2} P_{N-1, \alpha+\sigma, \beta+\theta}\left((z+1)^{2} \partial_{z} v(z)\right) d z+\xi
$$

where $\xi$ is chosen in such a way that $v(1)=\phi(1)$. By the projection theorem,

$$
\begin{aligned}
& \left\|\partial_{x}\left(\Pi_{N}^{1} v-v\right)\right\|_{\mu_{1}+\sigma_{1}, \mu_{2}+\sigma_{2}}+\left\|\Pi_{N}^{1} v-v\right\|_{\lambda_{1}+\sigma_{1}, \lambda_{2}+\sigma_{2}}+\left\|\Pi_{N}^{1} v-v\right\|_{\sigma_{1}, \sigma_{2}} \\
& \leq\left\|\partial_{x}(\phi-v)\right\|_{\mu_{1}+\sigma_{1}, \mu_{2}+\sigma_{2}}+\|\phi-v\|_{\lambda_{1}+\sigma_{1}, \lambda_{2}+\sigma_{2}}+\|\phi-v\|_{\sigma_{1}, \sigma_{2}} .
\end{aligned}
$$


Due to (3.7), a direct calculation with Theorem 2.3 leads to

$$
\left\|\partial_{x}(\phi-v)\right\|_{\mu_{1}+\sigma_{1}, \mu_{2}+\sigma_{2}}=\left\|\partial_{x}(\phi-v)\right\|_{\chi_{R}^{(\alpha, \beta)}} \leq c\left\|\partial_{x}(\phi-v)\right\|_{\chi_{R}^{(\alpha+\sigma-4, \beta+\theta)}} .
$$

Next, let $\gamma=-\sigma_{1}-\sigma_{2}-\lambda_{1}-\lambda_{2}-2$ and $\delta=\sigma_{1}+\lambda_{1}$. By (3.7), (3.8) and Lemma 2.2 ,

$$
\|\phi-v\|_{\lambda_{1}+\sigma_{1}, \lambda_{2}+\sigma_{2}}=\|\phi-v\|_{\chi_{R}^{(\gamma, \delta)}} \leq c\left\|\partial_{x}(\phi-v)\right\|_{\chi_{R}^{(\alpha+\sigma-4, \beta+\theta)}} .
$$

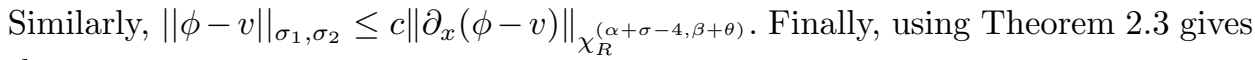
that

$$
\begin{aligned}
\left\|\partial_{x}(\phi-v)\right\|_{\chi_{R}^{(\alpha+\sigma-4, \beta+\theta)}} & =\left\|P_{N-1, \alpha+\sigma, \beta+\theta}\left((x+1)^{2} \partial_{x} v\right)-(x+1)^{2} \partial_{x} v\right\|_{\chi_{R}^{(\alpha+\sigma, \beta+\theta)}} \\
& \leq c N^{1-r}|v|_{r, \chi_{R}^{(\alpha+\sigma-1, \beta+\theta-1)}, A}=c N^{1-r}|v|_{B_{\mu_{1}, \mu_{2}, \sigma_{1}, \sigma_{2}, \sigma, \theta}^{r}}
\end{aligned}
$$

Then, the conclusion comes immediately from the previous statements.

\section{REFERENCES}

1. R. Askey, Orthogonal Polynomials and Special Functions, Regional Conference Series in Applied Mathematics, Vol. 21, SIAM, Philadelphia, 1975. MR0481145 (58:1288)

2. R. K. Bullough and P. J. Caudrey, The soliton and its history, in Solitons, ed. by R. K. Bullough and P. J. Caudrey, Springer-Verlag, Berlin, 1980. MR625877 (82m:35001)

3. J. Bergh and J. Löfström, Interpolation Spaces, An Introduction, Spinger-Verlag, Berlin, 1976. MR 0482275 (58:2349)

4. F. Black and M. Scholes, The pricing of options and corporate liabilities, J. of Political Economy, 81(1973), 637-654.

5. F. Black, E. Derman and W. Toy, A one factor model of interest rates and its application to treasury bond options, Financial Analysts Journal, 46(1990), 33-39.

6. J. P. Boyd, Spectral method using rational basis functions on an infinite interval, J. Comp. Phys., 69(1987), 112-142. MR892255 (88e:65093)

7. J. P. Boyd, Orthogonal rational functions on a semi-infinite interval, J. Comp. Phys., 70(1987), 63-88. MR888932 (88d:65034)

8. J. C. Cox, J. E. Ingersoll and S. A. Ross, A theory of the term structure of interest rates, Econometrica, 53(1985), 385-407. MR.785475

9. L. U. Dothan, On the term structure of interest rates, J. of Financial Economics, 6(1978), 59-69.

10. Guo Ben-yu, Jacobi approximations in certain Hilbert spaces and their applications to singular differential equations, J. Math. Anal. Appl., 243(2000), 373-408. MR.1741531(2001b:65082)

11. Ben-yu Guo and Jie Shen, On spectral approximations using modified Legendre rational functions: Application to the Korteweg de Vries equation on the half line, Indiana Uni. Math. J., 50(2001), 181-204. MR.1855668 (2002i:35165)

12. Ben-yu Guo and Jie Shen, Irrational approximations and their applications to partial differential equations in exterior domains, Adv. in Comp. Math., DOI 10.1007/s10444-006-9020-5.

13. Ben-yu Guo, Jie Shen and Zhong-qing Wang, A rational approximation and its applications to differential equations on the half line, J. of Sci. Comp., 15(2000), 117-148. MR.1827574 (2002a:41013)

14. Ben-yu Guo, Jie Shen and Zhong-qing Wang, Chebyshev rational spectral and pseudospectral methods on a semi-infinite interval, Int. J. Numer. Meth. Engng., 53 (2002), 65-84. MR 1870735 (2002k:65199)

15. Guo Ben-yu and Wang Li-lian, Jacobi interpolation approximations and their applications to singular differential equations, Advances in Computational Mathematics, 14(2001), 227-276. MR.1845244 (2002f:41003)

16. Guo Ben-yu and Wang Li-lian, Jacobi approximations in non-uniformly Jacobi-weighted Sobolev spaces. J. of Approximation. Theory, 128(2004), 1-41. MR2063010 (2005h:41010)

17. Guo Ben-yu and Wang Zhong-qing, Modified Chebyshev rational spectral method for the whole line, Discrete and Continuous Dynamical System, Supplement Volume, 2003, 365-374. MR 2018137 (2004k:65181) 
18. Guo Ben-yu and Wang Zhong-qing, Legendre rational approximation on the whole line, Science in China, Ser. A, Mathematics, 47(2004), Supp. 155-164. MR2079538 (2005f:41039)

19. G. H. Hardy, J. E. Littlewood and G. Pólya, Inequalities, Cambridge University Press, Cambridge, 1952. MR0046395 (13:727e)

20. O. A. Ladyzhenskaya, The Mathematical Theory of Viscous Incompressible Flow, English translation, Second edition, Gordon and Breach, New York, 1969. MR0254401 (40:7610)

21. P. J. Roache, Computational Fluid Dynamics, 2nd Edition, Hermosa Publishers, Albuquerque, 1976. MR 0411358 (53:15094)

22. Wang Zhong-qing and Guo Ben-yu, A rational approximation and its application to nonlinear differential equations on the whole line, J. Math. Anal. Appl., 247(2002), 374-403. MR.1936704 (2003g:41019)

23. Wang Zhong-qing and Guo Ben-yu, Modified Legendre rational spectral method for the whole line, J. Comp. Math., 22(2004), 457-474. MR2056301(2005c:65110)

24. Wang Zhong-qing and Guo Ben-yu, A mixed spectral method for incompressible viscous fluid flow in an infinite strip, Comp. Appl. Math., 24(2005), 343-364. MR2240448

Department of Mathematics, Division of Computational Science of E-institute of Shanghai Universities, Shanghai Normal University, Shanghai, 200234, People's RepubLIC OF CHINA

E-mail address: zqwang@shnu.edu.cn

Department of Mathematics, Division of Computational Science of E-institute of Shanghai Universities, Shanghai Normal University, Shanghai, 200234, People's RepubLIC OF CHINA

E-mail address: byguo@shnu.edu.cn 